\title{
On the large $\mathrm{R}$-charge $\mathcal{N}=2$ chiral correlators and the Toda equation
}

\author{
Matteo Beccaria \\ Dipartimento di Matematica e Fisica Ennio De Giorgi, Università del Salento \& INFN, \\ Via Arnesano, 73100 Lecce, Italy \\ E-mail: matteo.beccaria@le.infn.it
}

AbStraCt: We consider $\mathcal{N}=2 \mathrm{SU}(N)$ SQCD in four dimensions and a weak-coupling regime with large R-charge recently discussed in arXiv:1803.00580. If $\varphi$ denotes the adjoint scalar in the $\mathcal{N}=2$ vector multiplet, it has been shown that the 2-point functions in the sector of chiral primaries $\left(\operatorname{Tr} \varphi^{2}\right)^{n}$ admit a finite limit when $g_{\mathrm{YM}} \rightarrow 0$ with large R-charge growing like $\sim 1 / g_{\mathrm{YM}}^{2}$. The correction with respect to $\mathcal{N}=4$ correlators is a non-trivial function $F(\lambda ; N)$ of the fixed coupling $\lambda=n g_{\mathrm{YM}}^{2}$ and the gauge algebra rank $N$. We show how to exploit the Toda equation following from the $t t^{*}$ equations in order to control the R-charge dependence. This allows to determine $F(\lambda ; N)$ at order $\mathcal{O}\left(\lambda^{10}\right)$ for generic $N$, greatly extending previous results and placing on a firmer ground a conjecture proposed for the $\mathrm{SU}(2)$ case. We show that a similar Toda equation, discussed in the past, may indeed be used for the additional sector $\left(\operatorname{Tr} \varphi^{2}\right)^{n} \operatorname{Tr} \varphi^{3}$ due to the special mixing properties of these composite operators on the 4-sphere. We discuss the large R-limit in this second case and compute the associated scaling function $F$ at order $\mathcal{O}\left(\lambda^{7}\right)$ and generic $N$. Large $N$ factorization is also illustrated as a check of the computation.

KEYwords: Extended Supersymmetry, Matrix Models

ARXIV EPRINT: 1809.06280 


\section{Contents}

1 Introduction $\quad 1$

2 Chiral correlators in $\mathcal{N}=2$ superconformal theories $\quad 4$

2.1 Large R-charge limit of $\mathcal{N}=2$ correlators 6

3 Localization computation of the chiral correlators $\quad 7$

3.1 Matrix model $\mathcal{N}=2$ partition function 8

3.2 The $\mathcal{N}=4$ case and Gaussian correlators 8

$3.3 \mathcal{N}=2$ as a perturbation around $\mathcal{N}=4 \mathrm{SYM} \quad 9$

3.4 Sample 5-loops chiral two-point functions 9

4 Toda equation as a constraint: the function $F(\lambda ; N)$ at $\mathcal{O}\left(\lambda^{10}\right) \quad 12$

$\begin{array}{lll}4.1 & \text { Large R-charge limit } & 14\end{array}$

5 Decoupled Toda equation for the $\operatorname{Tr} \varphi^{3}$ tower $\quad 15$

$\begin{array}{lll}5.1 \text { Using the Toda equation as a constraint for the } \widehat{g}_{2 n} & 16\end{array}$

$\begin{array}{lll}5.2 & \text { Large R-charge limit } & 17\end{array}$

6 Relation with large $N$ factorization $\quad 18$

A Functions $F^{(\ell)}(N)$ for $\ell=8,9,10 \quad 19$

$\begin{array}{ll}\text { B The correlators } \widehat{g}_{2 n} \text { for } n=2,3 & 20\end{array}$

C Decoupling violations and Toda equation for general towers 21

\section{Introduction}

The search for explicit tests of AdS/CFT correspondence has often induced new applications of old non-perturbative theoretical tools aimed at connecting the two sides of the correspondence.

From the point of view of the dual gauge theory, superconformal invariance implies that natural objects to be addressed are 2- and 3-point functions of primary fields. In the early studies of type IIB superstring on $\mathrm{AdS}_{5} \times S_{5}$ dual to $\mathcal{N}=4 \mathrm{SYM}$ in $4 \mathrm{~d}$, particular attention has been devoted to the protected chiral sector where the supergravity limit is exact due to superconformal non-renormalization theorems [1-5]. Instead, more general unprotected operators have anomalous dimensions depending non-trivially on the 't Hooft coupling. Currently, they are efficiently computed by the Quantum Spectral Curve approach [6-8]. 
Wilson loops are a (non-local) different class of gauge theory observables which are also particularly suited from the point of view of testing AdS/CFT. In $\mathcal{N}=4 \mathrm{SYM}$, the relevant observable is the Maldacena-Wilson loop $W(C)$ where the six scalar fields are coupled to the loop $C$ in a locally supersymmetric way [9]. At strong coupling, $W(C)$ has a clean dual representation as a disc string amplitude. In some cases, exact results for $W(C)$ are available using supersymmetric localization [10]. Indeed, it was first conjectured that the $\frac{1}{2}$-BPS circular loop could have been captured by a matrix model $[11,12]$. Pestun proved this claim in [13] by showing that the path-integral computing $W(C)$ in $S^{4}$ can be obtained from the contributions at a finite set of critical points. This remarkable feature, due to supersymmetry, reduces the calculation to a finite dimensional (Gaussian) matrix model as first suggested in [11]. These exact results give strong coupling predictions that open the way to very non-trivial tests of the AdS/CFT correspondence, as in [14].

If supersymmetry is reduced to $\mathcal{N}=2$, where the low energy effective action is captured by Seiberg-Witten theory [15, 16], further exact results are available by exploiting once again localization [17]. Indeed, Pestun's analysis in [13] already includes the case of pure $\mathcal{N}=2$ and mass-deformed $\mathcal{N}=2^{*} \mathrm{SYM}$ on the 4 -sphere $S^{4}$. With respect to the $\mathcal{N}=4$ theory, there is an additional perturbative 1-loop contribution and instanton corrections encoded in Nekrasov partition function [18, 19]. The associated matrix model is no more Gaussian, but nevertheless may be treated efficiently. ${ }^{1}$

Here we are interested in high order perturbative expansion of correlators of chiral primaries in $\mathcal{N}=2$ superconformal theories in $4 \mathrm{~d}$. Although not protected as in $\mathcal{N}=4$, chiral primaries belong to short multiplets and are annihilated by all right-chiral supercharges. This implies that, under operator product expansion (OPE), they have a (chiral) ring structure whose dependence on the marginal gauge couplings is non-trivial, and encoded in the coefficients appearing in the 2- and 3-point functions. In particular, it has been shown how to compute the full set of chiral two-point functions in $\mathcal{N}=2$ superconformal $\mathrm{SU}(N)$ Yang-Mills theory with $N_{f}=2 N$ fundamental hypermultiplets by localization [24-27].

In this context, the authors of [28] have recently reconsidered the study of particular chiral correlators of the form

$$
g_{2 n}(\tau, \bar{\tau})=\left\langle\left(\operatorname{Tr} \varphi^{2}\right)^{n}\left(\operatorname{Tr} \bar{\varphi}^{2}\right)^{n}\right\rangle, \quad \varphi \equiv \varphi(\infty), \bar{\varphi} \equiv \bar{\varphi}(0),
$$

where $\tau=\frac{\theta}{2 \pi}+\frac{4 \pi i}{g_{\mathrm{YM}}^{2}}$ is the complexified gauge coupling and $\varphi$ is the complex adjoint scalar in the $\mathcal{N}=2$ vector multiplet. The 2-point function $g_{2 n}(\tau, \bar{\tau})$ may be computed in the large R-charge limit where ${ }^{2}$

$$
g \rightarrow 0, \quad \text { with fixed } \lambda=n g^{2} .
$$

In the large $n$ limit (1.2), it is possible to show that the following ratio is finite

$$
F(\lambda ; N)=\lim _{n \rightarrow \infty} \frac{\left.g_{2 n}\right|_{\mathrm{SQCD}}}{\left.g_{2 n}\right|_{\mathcal{N}=4}} .
$$

\footnotetext{
${ }^{1}$ For applications of localization to Wilson loops in $\mathcal{N}=2$ superconformal theories see [20-23].

${ }^{2}$ The fixed large $n$ coupling $\lambda$ in (1.2) follows the notation in [28] and should not be confused with the large $N$ 't Hooft coupling.
} 
The function $F(\lambda ; N)$ has certain special features discussed in [28], like universality and exponentiation in the $\mathrm{SU}(2)$ case, and seems an interesting non-trivial object by itself. It has been computed at order $\mathcal{O}\left(\lambda^{3}\right)$ for generic gauge group rank $N$, and at order $\mathcal{O}\left(\lambda^{5}\right)$ for the specific values $N=2,3,4,5$.

From a general perspective, it is interesting that non-trivial correlators are found in the zero coupling limit, as soon as the R-charge is large $\sim 1 / g^{2}$. Further motivation for the study of the limit (1.2) is that it falls into the general framework of semiclassical effective field theory description of strongly-coupled conformal theories in sectors of large global charge $[29,30] .^{3}$

As shown in [24], the correlator (1.1) obeys a Toda equation equivalent to the $4 \mathrm{~d}$ version of the two-dimensional topological-anti-topological fusion $t t^{*}$ equations $[43,44]$. In the analysis of [28], this fact is used as check of the localization computations. In this paper, we consider the higher order calculation of the function $F(\lambda ; N)$ for generic $N$. To this aim, we shall exploit the Toda equation as a strong constraint to control the exact R-charge dependence order by order in the weak coupling expansion. This approach will turn out to be quite effective for the calculation of the ratio (1.3) and its large R-charge limit. In particular, we shall present the expression of $F(\lambda ; N)$ at order $\mathcal{O}\left(\lambda^{10}\right)$, valid for generic $N$. As a byproduct, our results support the exponentiation property observed for the $\mathrm{SU}(2)$ theory in [28].

A further important issue concerns which type of correlators may generalize those in (1.1), while still admitting a sensible large R-charge limit in the regime (1.2). As we explained, it is quite important to control the R-charge dependence by a (possibly modified) Toda equation. For $\mathrm{SU}(N)$ theories this simple decoupling of the $t t^{*}$ equations has been discussed in $[25,26]$ for the correlators

$$
g_{2 n}^{\Phi}(\tau, \bar{\tau})=\left\langle\left(\operatorname{Tr} \varphi^{2}\right)^{n} \Phi\left(\operatorname{Tr} \bar{\varphi}^{2}\right)^{n} \bar{\Phi}\right\rangle,
$$

and expected to be valid for suitable chiral primaries $\Phi$ at 2-loops, but violated at 3loops and higher order in general [26]. This is related to the mixing pattern that the conformal anomaly induces when chiral primaries are mapped from $\mathbb{R}^{4}$ to the 4 -sphere $S^{4}$ - where localization is naturally worked out. Here, we show that the case $\Phi=\operatorname{Tr} \varphi^{3}$ is special by exploiting the normal ordering properties of the composites $\left(\operatorname{Tr} \varphi^{2}\right)^{n} \operatorname{Tr} \varphi^{3}$ and their relation with mixing [27]. We prove by explicit localization computations that the correlators in (1.4) obey in this case a modified decoupled Toda equation, first suggested in [25]. Besides, we exploit it to compute at order $\mathcal{O}\left(\lambda^{7}\right)$ and generic $N$ the function $F^{\Phi}$ expressing a ratio analogous to (1.3). Large $N$ factorization is also illustrated as a check of the computation.

It would be interesting to extend these calculations to other gauge algebras and to more complicated correlators like the one-point functions of chiral primaries in presence of Wilson loops, recently treated by localization in [45]. Besides, it would be desirable to understand what is the meaning of the proposed large R-charge limit from the AdS/CFT perspective, i.e. whether it has a useful dual gravity interpretation.

\footnotetext{
${ }^{3}$ Additional references about the large charge expansion of general conformal field theories and in AdS/CFT context may be found in [31-42].
} 
The detailed plan of the paper is the following. In section 2 we briefly recall the main definitions, previous results on chiral 2-point functions, and their large R-charge limit. In section 3 we introduce the basic tools needed to compute chiral correlators by localization, the map to the matrix model observables, and also give some explicit examples at 5-loop order. In section 4 we explain how to use the Toda equation to control the R-charge dependence in the $\left(\operatorname{Tr} \varphi^{2}\right)^{n}$ sector and derive our main result, i.e. the function $F(\lambda ; N)$ at order $\mathcal{O}\left(\lambda^{10}\right)$ and generic $N$. Finally, in section 5 we discuss the sector $\left(\operatorname{Tr} \varphi^{2}\right)^{n} \operatorname{Tr} \varphi^{3}$, illustrate a modified Toda equation and test it against low level explicit correlators, and obtain the associated large R-charge scaling function at order $\mathcal{O}\left(\lambda^{7}\right)$ and generic $N$. Section 6 is devoted to a brief discussion of the large $N$ expansion of our results. Various appendices collect technical details and further discussions.

\section{Chiral correlators in $\mathcal{N}=2$ superconformal theories}

Four dimensional superconformal $\mathcal{N}=2$ theories admit chiral primary operators $\Phi_{I}$ which are primary fields annihilated by half of the supersymmetry charges, $\left[\bar{Q}, \Phi_{I}\right]=0$. In terms of the R-symmetry group $\mathrm{SU}(2)_{R} \times \mathrm{U}(1)_{r}$, and dilatation eigenvalue $\Delta$, they have vanishing $\mathrm{SU}(2)_{R}$ isospin and abelian R-charge $r$ saturating the unitarity bound $\Delta=\frac{r}{2} \cdot{ }^{4}$ The 2-point function between chiral primaries is

$$
\left\langle\Phi_{I}(x) \bar{\Phi}_{\bar{J}}(0)\right\rangle=\frac{g_{I \bar{J}}}{|x|^{2 \Delta}}
$$

where the coefficient $g_{I \bar{J}}$ depends on the marginal couplings. The operator product expansion is non singular and reads [48]

$$
\Phi_{I}(x) \Phi_{J}(0)=C_{I J}^{K} \Phi_{K}(0)+\ldots,
$$

where the chiral ring coefficients $C^{K}{ }_{I J}$ enter the 3-point functions, as usual. If there are exactly marginal couplings, the space of marginal deformations has complex coordinates $\left(z_{i}, \bar{z}_{i}\right)$ and the infinitesimal change of the action $\delta S=\delta z \int d^{4} x \mathcal{O}_{i}(x)$ (plus a similar antiholomorphic part) preserves $\mathcal{N}=2$ supersymmetry if $\mathcal{O}_{i}$ are dimension 4 descendants of chiral primaries $\varphi_{i}$ with $r=4$. Their Zamolodchikov metric $g_{i \bar{\jmath}}$ appearing in (2.1) is Kähler with a potential given by the logarithm of the partition function on $S^{4}$ regulated in order to preserve the massive supersymmetry algebra $\operatorname{OSp}(2 \mid 4)$ [49]

$$
g_{i \bar{\jmath}}=\partial_{i} \partial_{\bar{\jmath}} \log Z_{S^{4}}
$$

If the partition function $Z_{S^{4}}$ may be computed by localization, this provides the Zamolodchikov metric and the 2-point function of the $\Delta=2$ chiral primaries.

This approach has been exploited in $[24,50]$ discussing mainly the example of SU(2) SQCD, i.e. YM with 4 fundamental hypermultiplets. In that case, the chiral ring has one

\footnotetext{
${ }^{4}$ Chiral primaries are usually assumed to be scalar fields with Lorentz spins $\left(j_{1}, j_{2}\right)=(0,0)$. Actually, one of the two Lorentz spin is automatically zero, while the other is zero in Lagrangian theories and expected to be zero in general, see [46]. In general, they can be detected from the superconformal index, see for instance the review paper [47].
} 
primary $\varphi_{2 n} \sim\left(\operatorname{Tr} \varphi^{2}\right)^{n}$ for each dimension $\Delta=2 n$, where $\varphi$ is the complex adjoint scalar in the $\mathcal{N}=2$ vector multiplet. Formula (2.3) may be fully extended to compute the 2-point function $g_{2 n}(\tau, \bar{\tau})$ in

$$
\left\langle\varphi_{2 n}(x) \bar{\varphi}_{2 n}(0)\right\rangle=\frac{g_{2 n}(\tau, \bar{\tau})}{|x|^{4 n}},
$$

where $\tau$ is the Yang-Mills complexified coupling

$$
\tau=\frac{\theta}{2 \pi}+\frac{4 \pi i}{g^{2}}
$$

To this aim, the $n=1$ input, taken from (2.3), is fed into the following (semi-infinite) Toda equation ${ }^{5}$

$$
\partial_{\tau} \partial_{\bar{\tau}} \log g_{2 n}=\frac{g_{2 n+2}}{g_{2 n}}-\frac{g_{2 n}}{g_{2 n-2}}-g_{2} .
$$

This equation is a special case of the $4 \mathrm{~d}$ analogue of the two-dimensional $t t^{*}$ topological fusion equations $[43,44]$ for the $\mathcal{N}=(2,2)$ chiral ring and derived in $4 \mathrm{~d}$ using superconformal Ward identities in [51]. ${ }^{6}$

These results have been extended in [25] to SU(N) SQCD, with $N_{f}=2 N$ massless fundamental hypermultiplets. The extension is not trivial because, altough there is again only one marginal coupling, the structure of the chiral ring is more complicated. In the $\mathrm{SU}(N)$ theory, a generic chiral primary takes the form

$$
\varphi_{\left\{n_{1}, \ldots, n_{N-1}\right\}} \sim \prod_{\ell=1}^{N-1}\left(\operatorname{Tr} \varphi^{\ell+1}\right)^{n_{\ell}} .
$$

The $t t^{*}$ equations are now an infinite set of coupled, non-linear equations for matrix-valued objects whose dimension increases with the conformal dimension. Due to the special nature of $\varphi_{2}=\operatorname{Tr} \varphi^{2}$, which is the only one with $\Delta=2$, it has been proposed that the $t t^{*}$ equations may be decoupled into separate Toda equations [25]. In particular this has been conjectured to happen for special primaries $\Phi$ - called $C_{2}$-primaries - connstructed such that the two point functions

$$
g_{2 n}^{\Phi}=\left\langle\Phi^{(n)}(1) \bar{\Phi}^{(n)}(0)\right\rangle, \quad \Phi^{(n)}=\left(\operatorname{Tr} \varphi^{2}\right)^{n} \Phi,
$$

obey the modified Toda equation, cf. (2.6),

$$
\partial_{\tau} \partial_{\bar{\tau}} \log g_{2 n}^{\Phi}=\frac{g_{2 n+2}^{\Phi}}{g_{2 n}^{\Phi}}-\frac{g_{2 n}^{\Phi}}{g_{2 n-2}^{\Phi}}-g_{2}
$$

Relation (2.9) was tested at 2 loops $^{7}$ in $\mathrm{SU}(3)$ and $\mathrm{SU}(4)$ theories by using conventional Feynman diagrams, see for instance [52-54]. In order to push the test to higher order, in [26] is has been shown how to use localization for the computation of rather general chiral 2point functions (and related extremal 3-point functions). ${ }^{8}$ For each chiral primary $\varphi_{i}$

\footnotetext{
${ }^{5}$ To be precise, (2.6) may be mapped to the Toda equation by a simple change of variable.

${ }^{6}$ Notice that the 2-point functions $g_{2 n}$ are enough to compute the extremal 3-point function $\left\langle\varphi_{2 m}\left(x_{1}\right) \varphi_{2 n}\left(x_{2}\right) \bar{\varphi}_{2 m+2 n}(y)\right\rangle$ due to the associativity properties of the chiral ring algebra.

${ }^{7}$ To be clear, this means the correction $\mathcal{O}\left(g_{\mathrm{YM}}^{4}\right)$ with respect to the tree level value.

${ }^{8}$ See also [55] for a discussion in terms of Alday-Gaiotto-Tachikawa duality.
} 
(marginal or not) it is possible to deform the partition function on $S^{4}$ by a term associated with the complex coupling $\tau_{i}$ such that

$$
\left\langle\varphi_{i}(\mathrm{~N}) \bar{\varphi}_{\bar{\jmath}}(\mathrm{S})\right\rangle_{S^{4}}=\partial_{\tau_{i}} \partial_{\bar{\tau}_{j}} \log Z_{S^{4}}(\boldsymbol{\tau}, \overline{\boldsymbol{\tau}}),
$$

where N,S are the north and south poles of $S^{4}$. Since $\mathcal{N}=2$ supersymmetry is preserved, it is possible to compute $\log Z_{S^{4}}(\boldsymbol{\tau}, \overline{\boldsymbol{\tau}})$ by minor modifications of the usual localization formulas at weak-coupling, especially when instanton corrections are not a concern as in our weak-coupling expansions.

A well known complication is that we are interested in flat space correlators, while (2.10) is computed on the 4-sphere and, due to conformal anomaly, there are important differences. This is expressed concisely in terms of a mixing

$$
\varphi_{\mathbb{R}^{4}} \rightarrow \varphi_{S^{4}}^{\Delta}+c_{1} R \varphi_{S^{4}}^{\Delta-2}+c_{2} R^{2} \varphi_{S^{4}}^{\Delta-4}+\ldots,
$$

where $R$ is the Ricci scalar. The explicit mixing coefficients may be found by a GramSchmidt orthogonalization of the operators on $S^{4} .{ }^{9}$ In general, this procedure leads to far from explicit results. One important exception is the case of $g_{2 n}$ in (2.4). In this case, in $[26]$ it is shown that $\left(Z \equiv Z_{S^{4}}\right)$

$$
g_{2 n}(\tau, \bar{\tau})=\frac{1}{Z} \frac{\operatorname{det} M^{(n)}}{\operatorname{det} M^{(n-1)}}, \quad M_{a b}^{(p)}=\partial_{\tau}^{a} \partial_{\bar{\tau}}^{b} Z, \quad a, b=0, \ldots, p .
$$

The ultimate reason for this remarkable determinant representation is the simple mixing pattern of the $\left(\operatorname{Tr} \varphi^{2}\right)^{n}$ operators for which $(2.11)$ reads

$$
\left(\operatorname{Tr} \varphi^{2}\right)^{n} \rightarrow\left(\operatorname{Tr} \varphi^{2}\right)^{n}+c_{1} R\left(\operatorname{Tr} \varphi^{2}\right)^{n-1}+c_{2} R^{2}\left(\operatorname{Tr} \varphi^{2}\right)^{n-2}+\cdots+c_{n} R^{n} \mathbb{I}
$$

and Gram-Schmidt orthogonalization is a triangular matrix in the one-dimensional vector space of chiral primaries. For our purposes, an important remark is that (2.12) implies the Toda equation (2.6) [26]. Explicit 3 loop calculations carried on in $\mathrm{SU}(3)$ and $\mathrm{SU}(4)$ theories in [26] suggests that the generalized relation (2.9) is not valid at all orders for the $C_{2}$ primaries introduced in [25], i.e. the $t t^{*}$ equations do not decouple. ${ }^{10}$

\section{$2.1 \quad$ Large $\mathrm{R}$-charge limit of $\mathcal{N}=2$ correlators}

As we mentioned in the Introduction, the authors of [28] have recently proposed to study the chiral correlators $g_{2 n}$ in (2.4) by considering the zero gauge coupling limit $g \rightarrow 0$ while

\footnotetext{
${ }^{9}$ In general, multiple trace operators are mixed with single trace operators, at finite rank $N$. A special solution at large $N$ is discussed in [56] and applied to check the chiral primary correlator with a Wilson loop computed in [57] with a 2-matrix model proposal. The large $N$ limit of correlators is also studied in $[58-60]$.

${ }^{10}$ To clarify what happens, let us recall that the construction in [25] is based on the chiral primary tower $\left(\operatorname{Tr} \varphi^{2}\right)^{n} \mathcal{O}_{I}$, where $\left\{\mathcal{O}_{I}\right\}$ is a basis for fixed dimension chiral primaries with definite orthogonality properties. Its construction requires a rotation in field space that happens to be coupling-dependent starting at three loops. This extra $\tau, \bar{\tau}$ dependence spoils the decoupling property. An alternative point of view will be illustrated in section 5 , see also appendix C.
} 
simultaneously increasing large R-charge $\sim 1 / g^{2}$, see (1.2) Working in SU(N) SQCD, they proved that in this limit one has

$$
g_{2 n}=F(\lambda ; N)\left(\frac{\lambda}{2 \pi e}\right)^{2 n} n^{\frac{N^{2}-1}{2}}+\ldots
$$

where $F(\lambda ; N)$ is the asymptotic ratio to the $\mathcal{N}=4$ correlators defined in (1.3). In the following discussion, it will be convenient to define the fixed $\mathrm{R}$-charge ratios

$$
F(g, n ; N)=\frac{\left.g_{2 n}\right|_{\mathrm{SQCD}}}{\left.g_{2 n}\right|_{\mathcal{N}=4}}, \quad F(\lambda ; N)=\lim _{n \rightarrow \infty} F(\sqrt{\lambda / n}, n ; N) .
$$

The perturbative expansion of $F(g, n ; N)$ is in integer inverse powers of

$$
\operatorname{Im} \tau=\frac{4 \pi}{g^{2}}
$$

One of the results of [28] are the explicit first two corrections

$$
\begin{aligned}
F(g, n ; N)= & 1-\frac{9 n\left(N^{2}+2 n-1\right)}{4 \pi^{2}(\operatorname{Im} \tau)^{2}} \zeta(3) \\
& +\frac{5 n\left(2 N^{2}-1\right)\left(3 N^{4}+(15 n-3) N^{2}+20 n^{2}-15 n+4\right.}{4 \pi^{3} N\left(N^{2}+3\right)(\operatorname{Im} \tau)^{3}} \zeta(5)+\ldots
\end{aligned}
$$

From this expression, we obtain in the limit (1.2), see (2.15),

$$
F(\lambda ; N)=1-\frac{9 \zeta(3)}{32 \pi^{4}} \lambda^{2}+\frac{25\left(2 N^{2}-1\right) \zeta(5)}{64 \pi^{6} N\left(N^{2}+3\right)} \lambda^{3}+\ldots
$$

Explicit results at order $\mathcal{O}\left(\lambda^{5}\right)$ are presented in [28] for the specialized cases $N=2,3,4,5$. At higher orders, the function $F$ contains products of $\zeta$-functions. Remarkably, for the $\mathrm{SU}(2)$ theory, one can write

$$
F(\lambda ; 2)=\exp \left(-\frac{9 \zeta(3)}{32 \pi^{4}} \lambda^{2}+\frac{25 \zeta(5)}{128 \pi^{6}} \lambda^{3}-\frac{2205 \zeta(7)}{16384 \pi^{8}} \lambda^{4}+\frac{3213 \zeta(9)}{32768 \pi^{10}} \lambda^{5}+\ldots\right),
$$

with only simple $\zeta$-functions in the exponent. This feature has been conjectured to hold at all orders in the small $\lambda$ expansion. For $N>2$, only terms involving $\zeta(3)$ are supposed to exponentiate, i.e. all dependence on the transcendental $\zeta(3)$ is captured by the first term in (2.19) and is independent on $N$, as follows from (2.17).

\section{Localization computation of the chiral correlators}

In this section, we briefly recall the available tools to evaluate chiral correlators by localization, the map to the matrix model observables, and also give some explicit examples for later purposes. 


\subsection{Matrix model $\mathcal{N}=2$ partition function}

Let us consider the $\mathcal{N}=2 \mathrm{SU}(N)$ Yang-Mills theory with $N_{f}=2 N$ fundamental hypermultiplets (SQCD). The celebrated partition function resulting from localization reads [10]

$$
Z_{\mathrm{SQCD}}=\int d^{N} a \delta\left(\sum_{n} a_{n}\right) \Delta(a) e^{-2 \pi \operatorname{Im} \tau \sum a_{i}^{2}}\left|Z_{1-\text { loop }}\right|^{2}\left|Z_{\text {inst }}\right|^{2},
$$

where $a_{n}$ are the eigenvalues of the traceless $N \times N$ matrix $a$ related to the vacuum expectation value of the adjoint scalar $\varphi$ in the $\mathcal{N}=2$ vector multiplet. The function $\Delta(a)=\prod_{n<m}\left(a_{n}-a_{m}\right)^{2}$ is the Vandermonde determinant. The 1-loop contribution to the partition function is

$$
\left|Z_{1-\text { loop }}\right|^{2}=\frac{\prod_{n<m} H\left(a_{n}-a_{m}\right)^{2}}{\prod_{n} H\left(a_{n}\right)^{2 N}}, \quad H(x)=\prod_{n=1}^{\infty}\left(1+\frac{x^{2}}{n^{2}}\right)^{n^{2}} e^{-\frac{x^{2}}{n}} .
$$

Finally, $Z_{\text {inst }}$ is the Nekrasov partition function $[18,19]$ evaluated with equivariant $\Omega$ deformation $\varepsilon_{1,2}$ parameters equal to the inverse radius of $S^{4}$. In the limit (1.2) this contribution may be neglected and we shall not discuss it further.

\subsection{The $\mathcal{N}=4$ case and Gaussian correlators}

The partition function of $\mathcal{N}=4 \mathrm{SYM}$ is obtained by removing the 1-loop and instanton terms and evaluates to the simple expression

$$
Z_{\mathcal{N}=4}=\frac{(2 \pi)^{\frac{N-1}{2}}}{\sqrt{N}} \frac{G(N+2)}{(4 \pi \operatorname{Im} \tau)^{\frac{N^{2}-1}{2}}},
$$

where $G(N)$ is the Barnes G-function. ${ }^{11}$ For our applications, closely following the neat analysis in $[27,45]$, it is convenient to rescale the matrix $a$ in order to put the classical part of the partition function in standard Gaussian form $e^{-\operatorname{Tr} a^{2}}$. This requires

$$
a \rightarrow\left(\frac{g^{2}}{8 \pi^{2}}\right)^{1 / 2} a
$$

Going to the basis $a=\sum_{\ell=1}^{N^{2}-1} a^{\ell} t^{\ell}$, with $\operatorname{Tr} t^{a}=0$ and $\operatorname{Tr}\left(t^{a} t^{b}\right)=\frac{1}{2} \delta^{a b}$, one can show that the relevant measure is flat $\prod_{n=1}^{N} d a_{n} \Delta(a)=\mathrm{c}_{N} \prod_{\ell=1}^{N^{2}-1} d a^{\ell}$. Thus, matrix model expectation values are computed as Gaussian averages $\langle F(a)\rangle=\int d a e^{-\operatorname{Tr} a^{2}} F(a)$ where the normalization $d a=\prod_{\ell=1}^{N^{2}-1} \frac{d a^{\ell}}{\sqrt{2 \pi}}$ is chosen in order to have $\langle 1\rangle=1$. Explicit calculations are performed by first applying Wick contractions to pair all $a$ 's. Then, contractions are computed by using the Gaussian matrix model relation $\left\langle a^{p} a^{q}\right\rangle=\delta^{p q}$. This makes a pair of $t^{\ell}$ appear and reduction is achieved by using standard $\mathrm{SU}(N)$ identitities [27]. With the definition

$$
t_{n_{1}, n_{2}, \ldots}=\left\langle\operatorname{Tr}\left(a^{n_{1}}\right) \operatorname{Tr}\left(a^{n_{2}}\right) \ldots\right\rangle
$$

\footnotetext{
${ }^{11}$ We remind that for integer $N$ one has $G(N+2)=\prod_{n=1}^{N} n$ !
} 
one finds up to level $\sum_{i} n_{i}=8$ the expressions

$$
\begin{array}{rlrl}
t_{2} & =\frac{N^{2}-1}{2}, & t_{2,2} & =\frac{N^{4}-1}{4} \\
t_{4} & =\frac{\left(N^{2}-1\right)\left(2 N^{2}-3\right)}{4 N}, & t_{6} & =\frac{5\left(N^{2}-1\right)\left(N^{4}-3 N^{2}+3\right)}{8 N^{2}}, \\
t_{3,3} & =\frac{3\left(N^{2}-1\right)\left(N^{2}-4\right)}{8 N}, & t_{4,2} & =\frac{\left(N^{2}-1\right)\left(N^{2}+3\right)\left(2 N^{2}-3\right)}{8 N}, \\
t_{2,2,2} & =\frac{\left(N^{4}-1\right)\left(N^{2}+3\right)}{8}, & t_{8} & =\frac{7\left(N^{2}-1\right)\left(2 N^{6}-8 N^{4}+15 N^{2}-15\right)}{16 N^{3}}, \\
t_{2,6} & =\frac{5\left(N^{2}-1\right)\left(N^{2}+5\right)\left(N^{4}-3 N^{2}+3\right)}{16 N^{2}}, \\
t_{4,4} & =\frac{\left(N^{2}-1\right)\left(4 N^{6}+20 N^{4}-99 N^{2}+135\right)}{16 N^{2}}, t_{3,5} & =\frac{15\left(N^{2}-4\right)\left(N^{2}-2\right)\left(N^{2}-1\right)}{16 N^{2}}, \\
t_{2,3,3} & =\frac{3\left(N^{2}-4\right)\left(N^{2}-1\right)\left(N^{2}+5\right)}{16 N}, & & =\frac{\left(N^{2}-1\right)\left(N^{2}+3\right)\left(N^{2}+5\right)\left(2 N^{2}-3\right)}{16 N}, \\
t_{2,2,2,2} & =\frac{\left(N^{4}-1\right)\left(N^{2}+3\right)\left(N^{2}+5\right)}{16} .
\end{array}
$$

\section{$3.3 \mathcal{N}=2$ as a perturbation around $\mathcal{N}=4 \mathrm{SYM}$}

As is well known, see for instance [56], it is very convenient to obtain the $\mathcal{N}=2$ correlators from the $\mathcal{N}=4$ ones by simply expanding (3.2) using

$$
\log H(x)=-\left(1+\gamma_{\mathrm{E}}\right) x^{2}-\sum_{\ell=2}^{\infty}(-1)^{\ell} \frac{\zeta(2 \ell-1)}{\ell} x^{2 \ell},
$$

and reconstructing products of higher order traces $\operatorname{Tr} a^{n}$ in the expansion. This procedure is straightforward and we find $\left|Z_{1-\text { loop }}\right|^{2}=e^{-S_{\text {int }}}$ with (see (3.5))

$$
\begin{aligned}
S_{\mathrm{int}}= & 3 \zeta(3) t_{2,2}-\frac{10}{3} \zeta(5)\left(3 t_{2,4}-2 t_{3,3}\right)+\frac{7}{2} \zeta(7)\left(4 t_{2,6}-8 t_{3,5}+5 t_{4,4}\right) \\
& -\frac{6}{5} \zeta(9)\left(15 t_{2,8}-40 t_{3,7}+70 t_{4,6}-42 t_{5,5}\right)+\ldots
\end{aligned}
$$

Taking into account relations like (3.6) and the rescaling in (3.4), this gives immediately the relation between partition functions

$$
\begin{aligned}
& Z_{\mathrm{SQCD}}=Z_{\mathcal{N}=4}\left[1-\frac{3\left(N^{4}-1\right) \zeta(3)}{16 \pi^{2}(\operatorname{Im} \tau)^{2}}+\frac{5\left(N^{4}-1\right)\left(2 N^{2}-1\right) \zeta(5)}{32 \pi^{3} N(\operatorname{Im} \tau)^{3}}\right. \\
& +\left(9\left(N^{2}+1\right)\left(N^{2}+3\right)\left(N^{2}+5\right) \zeta(3)^{2}-\frac{35}{N^{2}}\left(8 N^{6}+4 N^{4}-3 N^{2}+3\right) \zeta(7)\right) \frac{N^{2}-1}{512 \pi^{4}(\operatorname{Im} \tau)^{4}} \\
& +\left(-15\left(N^{2}+1\right)\left(N^{2}+5\right)\left(N^{2}+7\right)\left(2 N^{2}-1\right) \zeta(3) \zeta(5)\right. \\
& \left.\left.+\frac{21}{N^{2}}\left(26 N^{8}+28 N^{6}-3 N^{4}+6 N^{2}-9\right) \zeta(9)\right) \frac{N^{2}-1}{512 \pi^{5} N(\operatorname{Im} \tau)^{5}}+\ldots\right] .
\end{aligned}
$$

\subsection{Sample 5-loops chiral two-point functions}

Chiral primaries may be computed by taking into account (3.8) and the normalization (3.9). Let us give some examples at 5 -loop order. This will be useful in the following to explicitly 
test certain differential equations of Toda type. In this section, we consider the following chiral primaries on $\mathbb{R}^{4}$

$$
\mathcal{O}_{2}=\operatorname{Tr} \varphi^{2}, \quad \mathcal{O}_{2,2}=\left(\operatorname{Tr} \varphi^{2}\right)^{2}, \quad \mathcal{O}_{2,3}=\left(\operatorname{Tr} \varphi^{2}\right) \operatorname{Tr} \varphi^{3}, \ldots
$$

These are related to mixed operators on $S^{4}$ that we can express conveniently in terms of the matrix model multiple traces, see (2.11),

$$
\begin{aligned}
\mathcal{O}_{2} & \rightarrow t_{2}+c_{2 ; 0}(g) \mathbb{I}, \quad \mathcal{O}_{2,2} \rightarrow t_{2,2}+c_{2,2 ; 2}(g) t_{2}+c_{2,2 ; 0} \mathbb{I}, \\
\mathcal{O}_{2,3} & \rightarrow t_{2,3}+c_{2,3 ; 3}(g) t_{3} .
\end{aligned}
$$

Here $t_{\boldsymbol{n}}$ are as in (3.5) but we have made explicit the rescaling (3.4) to make $g$ appear explicitly in the mixing coefficients. These are determined by Gram-Schmidt orthogonalization. For instance, one has in the above

$$
\begin{aligned}
c_{2 ; 0}(g)= & -\frac{N^{2}-1}{2}\left[1-\frac{3 g^{4}\left(N^{2}+1\right) \zeta(3)}{64 \pi^{4}}+\frac{15 g^{6}\left(N^{2}+1\right)\left(2 N^{2}-1\right) \zeta(5)}{1024 \pi^{6} N}\right. \\
& +g^{8}\left(\frac{9\left(N^{2}+1\right)\left(N^{2}+2\right) \zeta(3)^{2}}{2048 \pi^{8}}-\frac{35\left(8 N^{6}+4 N^{4}-3 N^{2}+3\right) \zeta(7)}{16384 \pi^{8} N^{2}}\right) \\
& +g^{10}\left(\frac{105\left(26 N^{8}+28 N^{6}-3 N^{4}+6 N^{2}-9\right) \zeta(9)}{262144 \pi^{10} N^{3}}\right. \\
& \left.\left.-\frac{225\left(N^{2}+1\right)\left(N^{2}+3\right)\left(2 N^{2}-1\right) \zeta(3) \zeta(5)}{65536 \pi^{10} N}\right)+\mathcal{O}\left(g^{12}\right)\right]
\end{aligned}
$$

and similar expansions for the other coefficients, as in

$$
\begin{aligned}
c_{2,2 ; 2}(g)= & -\left(N^{2}+1\right)\left[1-\frac{3 g^{4}\left(N^{2}+5\right) \zeta(3)}{64 \pi^{4}}+\frac{15 g^{6}\left(N^{2}+11\right)\left(2 N^{2}-1\right) \zeta(5)}{1024 \pi^{6} N}\right. \\
& +g^{8}\left(\frac{9\left(N^{4}+12 N^{2}+29\right) \zeta(3)^{2}}{2048 \pi^{8}}-\frac{35\left(N^{2}+19\right)\left(8 N^{6}+4 N^{4}-3 N^{2}+3\right) \zeta(7)}{16384 \pi^{8} N^{2}\left(N^{2}+1\right)}\right) \\
& +g^{10}\left(\frac{105\left(N^{2}+29\right)\left(26 N^{8}+28 N^{6}-3 N^{4}+6 N^{2}-9\right) \zeta(9)}{262144 \pi^{10} N^{3}\left(N^{2}+1\right)}\right. \\
& \left.\left.-\frac{225\left(N^{2}+5\right)\left(N^{2}+15\right)\left(2 N^{2}-1\right) \zeta(3) \zeta(5)}{65536 \pi^{10} N}\right)+\mathcal{O}\left(g^{12}\right)\right], \\
c_{2,3 ; 3}(g)= & \left.-\frac{N^{2}+5\left[1-\frac{3 g^{4}\left(N^{2}+7\right) \zeta(3)}{64 \pi^{4}}+\frac{15 g^{6}\left(N^{2}-1\right)\left(2 N^{4}+45 N^{2}+105\right) \zeta(5)}{1024 \pi^{6} N\left(N^{2}+5\right)}\right.}{2048 \pi^{8}}-\frac{35\left(8 N^{8}+260 N^{6}+281 N^{4}-378 N^{2}+693\right) \zeta(7)}{16384 \pi^{8} N^{2}\left(N^{2}+5\right)}\right) \\
& +g^{8}\left(\frac{9\left(N^{2}+7\right)\left(N^{2}+8\right) \zeta(3)^{2}}{262144 \pi^{10} N^{3}\left(N^{2}+5\right)}\right) \\
& +g^{10}\left(\frac{105\left(N^{2}-1\right)\left(26 N^{8}+1180 N^{6}+3309 N^{4}+3096 N^{2}+3861\right) \zeta(9)}{65536 \pi^{10} N\left(N^{2}+5\right)}\right) \\
& \left.\left.-\frac{225\left(N^{2}-1\right)\left(N^{2}+9\right)\left(2 N^{4}+45 N^{2}+105\right) \zeta(3) \zeta(5)}{2}\right)+\mathcal{O}\left(g^{12}\right)\right] .
\end{aligned}
$$

After resolution of mixing, correlators can be computed by simply replacing the r.h.s. in (3.11) and using the Gaussian correlators, cf. (3.6). Since we want to match the normalization in [26] for the 2-point functions of the form $\left\langle\mathcal{O}_{n}(\infty) \overline{\mathcal{O}}_{n}(0)\right\rangle$, we also have to multiply the matrix model expression by $2^{|\boldsymbol{n}|}=2^{n_{1}+n_{2}+\ldots}$ including also the factor $\left(\frac{g^{2}}{4 \pi}\right)^{|\boldsymbol{n}|}$. 
In particular, let us present the complete five-loop expressions (with generic $N$ ) of two correlators computed at 3-loops in [26] for $N=3$ and $N=4$. These are

$$
\begin{aligned}
& \left\langle\mathcal{O}_{2,2} \overline{\mathcal{O}}_{2,2}\right\rangle=8\left(N^{4}-1\right)\left(\frac{g^{2}}{4 \pi}\right)^{4}\left[1-\frac{9 g^{4}\left(N^{2}+3\right) \zeta(3)}{32 \pi^{4}}+\frac{15 g^{6}\left(N^{2}+6\right)\left(2 N^{2}-1\right) \zeta(5)}{128 \pi^{6} N}\right. \\
& +g^{8}\left(\frac{9\left(29 N^{4}+204 N^{2}+355\right) \zeta(3)^{2}}{4096 \pi^{8}}-\frac{175\left(N^{2}+10\right)\left(8 N^{6}+4 N^{4}-3 N^{2}+3\right) \zeta(7)}{8192 \pi^{8} N^{2}\left(N^{2}+1\right)}\right) \\
& +g^{10}\left(\frac{315\left(N^{2}+15\right)\left(26 N^{8}+28 N^{6}-3 N^{4}+6 N^{2}-9\right) \zeta(9)}{65536 \pi^{10} N^{3}\left(N^{2}+1\right)}\right. \\
& \left.\left.-\frac{135\left(2 N^{2}-1\right)\left(7 N^{4}+78 N^{2}+211\right) \zeta(3) \zeta(5)}{16384 \pi^{10} N}\right)+\mathcal{O}\left(g^{12}\right)\right]
\end{aligned}
$$

and

$$
\begin{aligned}
\left\langle\mathcal{O}_{2,3} \overline{\mathcal{O}}_{2,3}\right\rangle= & 6 \frac{\left(N^{2}-1\right)\left(N^{2}-4\right)\left(N^{2}+5\right)}{N}\left(\frac{g^{2}}{4 \pi}\right)^{5}\left[1-\frac{9 g^{4}\left(N^{2}+5\right) \zeta(3)}{32 \pi^{4}}\right. \\
& +\frac{5 g^{6}\left(N^{2}-1\right)\left(46 N^{4}+703 N^{2}+1525\right) \zeta(5)}{1024 \pi^{6} N\left(N^{2}+5\right)}+g^{8}\left(\frac{9\left(7 N^{4}+75 N^{2}+215\right) \zeta(3)^{2}}{1024 \pi^{8}}\right. \\
& \left.-\frac{35\left(73 N^{8}+1501 N^{6}+1537 N^{4}-2043 N^{2}+3900\right) \zeta(7)}{16384 \pi^{8} N^{2}\left(N^{2}+5\right)}\right) \\
& +g^{10}\left(\frac{63(N-1)(N+1)\left(452 N^{8}+13312 N^{6}+36365 N^{4}+34180 N^{2}+41835\right) \zeta(9)}{262144 \pi^{10} N^{3}\left(N^{2}+5\right)}\right. \\
& \left.\left.-\frac{45(N-1)(N+1)\left(78 N^{6}+1721 N^{4}+11042 N^{2}+18575\right) \zeta(3) \zeta(5)}{32768 \pi^{10} N\left(N^{2}+5\right)}\right)+\mathcal{O}\left(g^{12}\right)\right] .
\end{aligned}
$$

Specialization to $\mathrm{SU}(3)$ gives

$$
\begin{aligned}
\left\langle\mathcal{O}_{2,2} \overline{\mathcal{O}}_{2,2}\right\rangle_{N=3}=\left(\frac{g^{2}}{4 \pi}\right)^{4}[ & 640-\frac{2160 g^{4} \zeta(3)}{\pi^{4}}+\frac{6375 g^{6} \zeta(5)}{\pi^{6}}+g^{8}\left(\frac{51075 \zeta(3)^{2}}{8 \pi^{8}}-\frac{1699075 \zeta(7)}{96 \pi^{8}}\right) \\
& \left.+g^{10}\left(\frac{417375 \zeta(9)}{8 \pi^{10}}-\frac{707625 \zeta(3) \zeta(5)}{16 \pi^{10}}\right)+\mathcal{O}\left(g^{12}\right)\right], \\
\left\langle\mathcal{O}_{2,3} \overline{\mathcal{O}}_{2,3}\right\rangle_{N=3}=\left(\frac{g^{2}}{4 \pi}\right)^{5}[ & 1120-\frac{4410 g^{4} \zeta(3)}{\pi^{4}}+\frac{144725 g^{6} \zeta(5)}{12 \pi^{6}}+g^{8}\left(\frac{458955 \zeta(3)^{2}}{32 \pi^{8}}-\frac{12273275 \zeta(7)}{384 \pi^{8}}\right) \\
& \left.+g^{10}\left(\frac{11641175 \zeta(9)}{128 \pi^{10}}-\frac{2945775 \zeta(3) \zeta(5)}{32 \pi^{10}}\right)+\mathcal{O}\left(g^{12}\right)\right],
\end{aligned}
$$

extending the 3-loops results in eq. (3.45) and eq. (3.56, first line) of [26]. Similarly, specialization to $\mathrm{SU}(4)$ gives

$$
\begin{aligned}
\left\langle\mathcal{O}_{2,2} \overline{\mathcal{O}}_{2,2}\right\rangle_{N=4}= & \left(\frac{g^{2}}{4 \pi}\right)^{4}\left[2040-\frac{43605 g^{4} \zeta(3)}{4 \pi^{4}}+\frac{1304325 g^{6} \zeta(5)}{32 \pi^{6}}\right. \\
& +g^{8}\left(\frac{25343685 \zeta(3)^{2}}{512 \pi^{8}}-\frac{1151616375 \zeta(7)}{8192 \pi^{8}}\right) \\
& \left.+g^{10}\left(\frac{266283200925 \zeta(9)}{524288 \pi^{10}}-\frac{3469385925 \zeta(3) \zeta(5)}{8192 \pi^{10}}\right)+\mathcal{O}\left(g^{12}\right)\right]
\end{aligned}
$$




$$
\begin{aligned}
\left\langle\mathcal{O}_{2,3} \overline{\mathcal{O}}_{2,3}\right\rangle_{N=4}= & \left(\frac{g^{2}}{4 \pi}\right)^{5}\left[5670-\frac{535815 g^{4} \zeta(3)}{16 \pi^{4}}+\frac{248558625 g^{6} \zeta(5)}{2048 \pi^{6}}\right. \\
& +g^{8}\left(\frac{81826605 \zeta(3)^{2}}{512 \pi^{8}}-\frac{13344472575 \zeta(7)}{32768 \pi^{8}}\right) \\
& \left.+g^{10}\left(\frac{11997966800925 \zeta(9)}{8388608 \pi^{10}}-\frac{87052714875 \zeta(3) \zeta(5)}{65536 \pi^{10}}\right)+\mathcal{O}\left(g^{12}\right)\right],
\end{aligned}
$$

extending the 3-loops results in eq. (3.46) and eq. (3.57) of [26].

\section{Toda equation as a constraint: the function $F(\lambda ; N)$ at $\mathcal{O}\left(\lambda^{10}\right)$}

The explicit results derived in the previous sections are not useful for the study of the large $n$ limit of $g_{2 n}$. Indeed, the computational complexity grows quickly with $n$, and an analytic treatment of the $n$-dependence is mandatory. We now show that this is fully provided by the Toda equation. The tree level value of $g_{2 n}$ is equal to its $\mathcal{N}=4$ limit and reads [56]

$$
\left.g_{2 n}\right|_{\mathcal{N}=4}=\frac{n ! 2^{2 n}}{(\operatorname{Im} \tau)^{2 n}} \frac{\Gamma\left(\frac{N^{2}-1}{2}+n\right)}{\Gamma\left(\frac{N^{2}-1}{2}\right)} .
$$

Explicit results, as in (2.17), suggest that the perturbative corrections takes the following form

$$
\begin{aligned}
\left.g_{2 n}\right|_{\mathrm{SQCD}}= & \frac{n ! 2^{2 n}}{(\operatorname{Im} \tau)^{2 n}} \frac{\Gamma\left(\frac{N^{2}-1}{2}+n\right)}{\Gamma\left(\frac{N^{2}-1}{2}\right)}\left[1+\mathcal{A}_{2} n \frac{n+\xi_{2,0}}{(\operatorname{Im} \tau)^{2}}+\mathcal{A}_{3} n \frac{n^{2}+\xi_{3,1} n+\xi_{3,0}}{(\operatorname{Im} \tau)^{3}}+\right. \\
& \left.+\mathcal{A}_{4} n \frac{n^{3}+\xi_{4,2} n^{2}+\xi_{4,1} n+\xi_{4,0}}{(\operatorname{Im} \tau)^{4}}+\ldots\right]
\end{aligned}
$$

where the constants $\mathcal{A}_{k}$ and $\xi_{p, q}$ are functions of $N$, but not of $n$ or the coupling. This simple structure may be plugged in the Toda equation (2.6). Remarkably, the Toda equation is strong enough to fix all these constants $\xi_{p, q}$ while the normalization $\mathcal{A}_{k}$ remains free. For instance, the first two corrections in (4.2) take the form

$$
\begin{aligned}
\left.g_{2 n}\right|_{\mathrm{SQCD}}= & \frac{n ! 2^{2 n}}{(\operatorname{Im} \tau)^{2 n}} \frac{\Gamma\left(\frac{N^{2}-1}{2}+n\right)}{\Gamma\left(\frac{N^{2}-1}{2}\right)}\left[1+\mathcal{A}_{2} n \frac{n+\frac{N^{2}-1}{2}}{(\operatorname{Im} \tau)^{2}}\right. \\
& \left.+\mathcal{A}_{3} n \frac{n^{2}+\frac{3}{4}\left(N^{2}-1\right) n+\frac{1}{20}\left(3 N^{4}-3 N^{2}+4\right)}{(\operatorname{Im} \tau)^{3}}+\ldots\right] .
\end{aligned}
$$

The Toda equation may be imposed at high order in the perturbative expansion. In general, the coefficients $\xi_{p, q}$ have also a dependence on the constants $\mathcal{A}_{k}$. For instance, at the next order, one finds

$$
\begin{aligned}
& \xi_{4,2}=N^{2}-1+\frac{\mathcal{A}_{2}^{2}}{\mathcal{A}_{4}} \\
& \xi_{4,1}=\frac{1}{28}\left(23-12 N^{2}+9 N^{4}-\left(20-20 N^{2}+N^{4}\right) \frac{\mathcal{A}_{2}^{2}}{\mathcal{A}_{4}}\right) \\
& \xi_{4,0}=\frac{1}{504}\left(18\left(-8+7 N^{2}+N^{6}\right)+\left(163-147 N^{2}+77 N^{4}-9 N^{6}\right) \frac{\mathcal{A}_{2}^{2}}{\mathcal{A}_{4}}\right) .
\end{aligned}
$$


The final expression may be evaluated at $n=1$ in terms of the correlator $g_{2}$ which is related to the double derivative of the partition function. This allows to fix the constants $\mathcal{A}_{k}$. Explicitly, one finds the following first five values ${ }^{12}$

$$
\begin{aligned}
\mathcal{A}_{2}= & -\frac{9 \zeta(3)}{2 \pi^{2}}, \quad \mathcal{A}_{3}=\frac{25\left(2 N^{2}-1\right) \zeta(5)}{\pi^{3} N\left(N^{2}+3\right)}, \\
\mathcal{A}_{4}= & \frac{81 \zeta(3)^{2}}{8 \pi^{4}}-\frac{1225\left(8 N^{6}+4 N^{4}-3 N^{2}+3\right) \zeta(7)}{16 \pi^{4} N^{2}\left(N^{2}+1\right)\left(N^{2}+3\right)\left(N^{2}+5\right)}, \\
\mathcal{A}_{5}= & \frac{1323\left(26 N^{8}+28 N^{6}-3 N^{4}+6 N^{2}-9\right) \zeta(9)}{4 \pi^{5} N^{3}\left(N^{2}+1\right)\left(N^{2}+3\right)\left(N^{2}+5\right)\left(N^{2}+7\right)}-\frac{225\left(2 N^{2}-1\right) \zeta(3) \zeta(5)}{2 \pi^{5} N\left(N^{2}+3\right)}, \\
\mathcal{A}_{6}= & \frac{11025\left(8 N^{6}+4 N^{4}-3 N^{2}+3\right) \zeta(3) \zeta(7)}{32 \pi^{6} N^{2}\left(N^{2}+1\right)\left(N^{2}+3\right)\left(N^{2}+5\right)} \\
& -\frac{17787\left(122 N^{10}+280 N^{8}+48 N^{6}-15 N^{4}+45\right) \zeta(11)}{16 \pi^{6} N^{4}\left(N^{2}+1\right)\left(N^{2}+3\right)\left(N^{2}+5\right)\left(N^{2}+7\right)\left(N^{2}+9\right)} \\
& +\frac{25\left(100 N^{12}+2331 N^{10}+13070 N^{8}+20941 N^{6}+20985 N^{4}+14138 N^{2}+7875\right) \zeta(5)^{2}}{2 \pi^{6} N^{2}\left(N^{2}+1\right)\left(N^{2}+3\right)^{2}\left(N^{2}+5\right)\left(N^{2}+7\right)\left(N^{2}+9\right)} \\
& -\frac{243 \zeta(3)^{3}}{16 \pi^{6}} .
\end{aligned}
$$

The full expression of $F(g, n ; N)$ is clearly unwieldy. With the definition

$$
F(g, n ; N)=1+\sum_{k=2}^{\infty} \frac{f_{k}(n ; N)}{(\operatorname{Im} \tau)^{k}}
$$

the $k=2,3$ terms have been written in (2.17). The next two are

$$
\begin{aligned}
f_{4}(n ; N)= & \frac{9 n\left(13-45 n+36 n^{2}+36 n^{3}+12\left(-1+3 n+3 n^{2}\right) N^{2}+(11+9 n) N^{4}\right) \zeta(3)^{2}}{32 \pi^{4}} \\
& -\frac{175 n\left(8-7 n+14 n^{2}+(1+7 n) N^{2}+N^{4}\right)\left(3-3 N^{2}+4 N^{4}+8 N^{6}\right) \zeta(7)}{64 \pi^{4} N^{2}\left(N^{2}+1\right)\left(N^{2}+3\right)\left(N^{2}+5\right)}, \\
f_{5}(n ; N)= & -\frac{45 n\left(-1+2 n+N^{2}\right)\left(-1+2 N^{2}\right)}{32 \pi^{5} N\left(N^{2}+3\right)}\left[57-52 n+90 n^{2}+40 n^{3}\right. \\
& \left.+6\left(1+9 n+5 n^{2}\right) N^{2}+3(3+2 n) N^{4}\right] \zeta(3) \zeta(5) \\
& \frac{21 n}{128 \pi^{5} N^{3}\left(N^{2}+1\right)\left(N^{2}+3\right)\left(N^{2}+5\right)\left(N^{2}+7\right)} \\
& \times\left[7\left(67-330 n+560 n^{2}-360 n^{3}+288 n^{4}\right)+30\left(-17+77 n-56 n^{2}+84 n^{3}\right) N^{2}\right. \\
& \left.+10\left(38-21 n+112 n^{2}\right) N^{4}+30(1+7 n) N^{6}+15 N^{8}\right] \zeta(9) .
\end{aligned}
$$

Additional terms are increasingly more involved, but with roughly the same structure. At this level, we did not identify any simple regularity.

\footnotetext{
${ }^{12}$ Although algorithmic, the procedure requires some careful coding when pushed to high orders. The complete solutions at order $\mathcal{O}\left(g^{20}\right)$ is available under request.
} 


\subsection{Large R-charge limit}

From our results for the functions $f_{k}$ in (4.6), we can take the limit (1.2). It is convenient to present the result in the following logarithmic form ${ }^{13}$ in order to emphasize possible exponentiation properties

$$
\log F(\lambda ; N)=\sum_{\ell=2}^{\infty}\left(\frac{\lambda}{8 \pi^{2}}\right)^{\ell} \sum_{s} F_{s}^{(\ell)}(N) \zeta(s), \quad \zeta(s)=\zeta\left(s_{1}\right) \zeta\left(s_{2}\right) \ldots
$$

At order $\mathcal{O}\left(\lambda^{10}\right)$, the functions $F_{\boldsymbol{s}}^{(\ell)}(N)$ are

$$
\begin{aligned}
& F_{3}^{(2)}(N)=-18, \\
& F_{5}^{(3)}(N)=\frac{200\left(2 N^{2}-1\right)}{N\left(N^{2}+3\right)}, \\
& F_{7}^{(4)}(N)=-\frac{1225\left(8 N^{6}+4 N^{4}-3 N^{2}+3\right)}{N^{2}\left(N^{2}+1\right)\left(N^{2}+3\right)\left(N^{2}+5\right)}, \\
& F_{9}^{(5)}(N)=\frac{10584\left(26 N^{8}+28 N^{6}-3 N^{4}+6 N^{2}-9\right)}{N^{3}\left(N^{2}+1\right)\left(N^{2}+3\right)\left(N^{2}+5\right)\left(N^{2}+7\right)}, \\
& F_{5^{2}}^{(6)}(N)=\frac{184800\left(N^{2}-4\right)\left(N^{6}-N^{4}-43 N^{2}-37\right)}{\left(N^{2}+1\right)\left(N^{2}+3\right)^{2}\left(N^{2}+5\right)\left(N^{2}+7\right)\left(N^{2}+9\right)}, \\
& F_{11}^{(6)}(N)=-\frac{71148\left(122 N^{10}+280 N^{8}+48 N^{6}-15 N^{4}+45\right)}{N^{4}\left(N^{2}+1\right)\left(N^{2}+3\right)\left(N^{2}+5\right)\left(N^{2}+7\right)\left(N^{2}+9\right)} . \\
& F_{5,7}^{(7)}(N)=-\frac{960960\left(N^{2}-4\right)\left(22 N^{8}+11 N^{6}-1167 N^{4}-531 N^{2}+705\right)}{N\left(N^{2}+1\right)\left(N^{2}+3\right)^{2}\left(N^{2}+5\right)\left(N^{2}+7\right)\left(N^{2}+9\right)\left(N^{2}+11\right)}, \\
& F_{13}^{(7)}(N)=\frac{8833968\left(34 N^{10}+110 N^{8}-29 N^{6}+20 N^{4}-15\right)}{N^{5}\left(N^{2}+3\right)\left(N^{2}+5\right)\left(N^{2}+7\right)\left(N^{2}+9\right)\left(N^{2}+11\right)},
\end{aligned}
$$

with the $\ell=8,9$, 10 functions being written in appendix A. One can check that all terms associated with multiple products of zeta functions vanish for $N=2$. For this value, our expression reduces to

$$
\begin{aligned}
\log F(\lambda ; 2)= & -\frac{9 \lambda^{2} \zeta(3)}{32 \pi^{4}}+\frac{25 \lambda^{3} \zeta(5)}{128 \pi^{6}}-\frac{2205 \lambda^{4} \zeta(7)}{16384 \pi^{8}}+\frac{3213 \lambda^{5} \zeta(9)}{32768 \pi^{10}} \\
& -\frac{78771 \lambda^{6} \zeta(11)}{1048576 \pi^{12}}+\frac{250965 \lambda^{7} \zeta(13)}{4194304 \pi^{14}}-\frac{105424605 \lambda^{8} \zeta(15)}{2147483648 \pi^{16}} \\
& +\frac{265525975 \lambda^{9} \zeta(17)}{6442450944 \pi^{18}}-\frac{12108123027 \lambda^{10} \zeta(19)}{343597383680 \pi^{20}}+\mathcal{O}\left(\lambda^{11}\right) .
\end{aligned}
$$

Extending the $\mathcal{O}\left(\lambda^{5}\right)$ results of [28], see (2.19), and confirming their conjecture for the exponentiation in terms of simple $\zeta$-functions, at least at this order. Other instances have

\footnotetext{
${ }^{13}$ Here $\boldsymbol{s}=\left(s_{1}, s_{2}, \ldots\right)$ is a multi-index with non-negative integer values. We shall also adopt the notation $s_{k}^{p}$ to denote repetitions of $p$ instances of $s_{k}$. So, for instance, $\zeta\left(3^{2}, 5^{3}\right)=\zeta(3,3,5,5,5)=\zeta(3)^{2} \zeta(5)^{3}$ and so on.
} 
a more complicated structure with products of $\zeta$-functions. For instance, in $\mathrm{SU}(3)$ one has

$$
\begin{aligned}
\log F(\lambda ; 3)= & -\frac{9 \lambda^{2} \zeta(3)}{32 \pi^{4}}+\frac{425 \lambda^{3} \zeta(5)}{2304 \pi^{6}}-\frac{17885 \lambda^{4} \zeta(7)}{147456 \pi^{8}}+\frac{5565 \lambda^{5} \zeta(9)}{65536 \pi^{10}} \\
& +\lambda^{6}\left(\frac{1925 \zeta(5)^{2}}{14155776 \pi^{12}}-\frac{2668897 \zeta(11)}{42467328 \pi^{12}}\right)+\lambda^{7}\left(\frac{32984237 \zeta(13)}{679477248 \pi^{14}}-\frac{5005 \zeta(5) \zeta(7)}{14155776 \pi^{14}}\right) \\
& +\lambda^{8}\left(\frac{35035 \zeta(7)^{2}}{150994944 \pi^{16}}+\frac{146575 \zeta(5) \zeta(9)}{402653184 \pi^{16}}-\frac{2245755655 \zeta(15)}{57982058496 \pi^{16}}\right) \\
& +\lambda^{9}\left(-\frac{1519375 \zeta(5)^{3}}{1174136684544 \pi^{18}}-\frac{3488485 \zeta(7) \zeta(9)}{7247757312 \pi^{18}}-\frac{546184925 \zeta(5) \zeta(11)}{1565515579392 \pi^{18}}\right. \\
& \left.+\frac{669686057755 \zeta(17)}{21134460321792 \pi^{18}}\right)+\lambda^{10}\left(\frac{8083075 \zeta(5)^{2} \zeta(7)}{1565515579392 \pi^{20}}+\frac{77643709 \zeta(9)^{2}}{309237645312 \pi^{20}}\right. \\
& \left.+\frac{2905703801 \zeta(7) \zeta(11)}{6262062317568 \pi^{20}}+\frac{4074100745 \zeta(5) \zeta(13)}{12524124635136 \pi^{20}}-\frac{29805018472801 \zeta(19)}{1127171217162240 \pi^{20}}\right)+\mathcal{O}\left(\lambda^{11}\right) .
\end{aligned}
$$

\section{Decoupled Toda equation for the $\operatorname{Tr} \varphi^{3}$ tower}

Given the effectiveness of Toda equation in the high order calculation of $g_{2 n}$, it is natural to ask whether similar cases may be treated with the same approach. As we explained, the Toda equation is a consequence of the determinant representation (2.12). On the other hand, this follows from the one-dimensional mixing structure of $\left(\operatorname{Tr} \varphi^{2}\right)^{n}$ in (2.13). From this point of view, a special case is that of $g_{2 n}^{\Phi}$, see (2.8), where $\Phi=\operatorname{Tr} \varphi^{3}$. To simplify notation, we shall denote this 2-point function by the special notation $\widehat{g}_{2 n}$.

The important (easy) remark is that $\left(\operatorname{Tr} \varphi^{2}\right)^{n} \operatorname{Tr} \varphi^{3}$ can only mix with similar operators with $n^{\prime}<n$. This is clear from the normal-ordering interpretation of mixing discussed in [27] and relations like (pairing stands for Wick contraction)

$$
\operatorname{Tr} \varphi^{2} \operatorname{Tr} \varphi^{3} \sim \operatorname{Tr} \varphi^{3}, \quad \operatorname{Tr}(\sqcap \varphi \varphi) \sim \operatorname{Tr} \varphi=0, \text { etc. }
$$

This simple mixing pattern implies the validity of the Toda equation (2.9), i.e.

$$
\partial_{\tau} \partial_{\bar{\tau}} \log \widehat{g}_{2 n}=\frac{\widehat{g}_{2 n+2}}{\widehat{g}_{2 n}}-\frac{\widehat{g}_{2 n}}{\widehat{g}_{2 n-2}}-g_{2} .
$$

Before showing how to use (5.2) to constrain the R-charge dependence, let us begin with some explicit check of (5.2) at low values of $n$. The correlator $\widehat{g}_{0}=\left\langle\operatorname{Tr} \varphi^{3} \operatorname{Tr} \bar{\varphi}^{3}\right\rangle$ is the easiest since there is no mixing to account for. One finds, at order $\mathcal{O}\left(g^{10}\right)$,

$$
\begin{aligned}
\widehat{g}_{0}= & \frac{3\left(N^{2}-4\right)\left(N^{2}-1\right)}{N}\left(\frac{g^{2}}{4 \pi}\right)^{3}\left[1-\frac{9 g^{4}\left(N^{2}+3\right) \zeta(3)}{64 \pi^{4}}+\frac{5 g^{6}\left(N^{2}-1\right)\left(22 N^{2}+53\right) \zeta(5)}{1024 \pi^{6} N}\right. \\
& +g^{8}\left(\frac{27\left(3 N^{4}+20 N^{2}+37\right) \zeta(3)^{2}}{4096 \pi^{8}}-\frac{105\left(11 N^{6}+12 N^{4}-16 N^{2}+29\right) \zeta(7)}{16384 \pi^{8} N^{2}}\right) \\
& +g^{10}\left(\frac{63\left(N^{2}-1\right)\left(192 N^{6}+552 N^{4}+515 N^{2}+645\right) \zeta(9)}{262144 \pi^{10} N^{3}}\right. \\
& \left.\left.-\frac{45\left(N^{2}-1\right)\left(50 N^{4}+373 N^{2}+633\right) \zeta(3) \zeta(5)}{65536 \pi^{10} N}\right)+\mathcal{O}\left(g^{12}\right)\right]
\end{aligned}
$$


The next correlator is

$$
\widehat{g}_{2}=\left\langle\operatorname{Tr} \varphi^{2} \operatorname{Tr} \varphi^{3} \operatorname{Tr} \bar{\varphi}^{2} \operatorname{Tr} \bar{\varphi}^{-3}\right\rangle,
$$

and has been computed in (3.15). Working out the mixing coefficients appearing in the correlators for $n=2,3$ one finds the explicit results collected in appendix B. Plugging these results in (5.2) and taking $g_{2}$ from (4.1) and (4.6), one checks that the Toda equation (5.2) for $\widehat{g}_{2 n}$ is indeed satisfied at this order.

The case of more complicated towers has roused some debate in the past. A detailed account of what happens to decoupling is discussed and clarified in a simple example in appendix C.

\subsection{Using the Toda equation as a constraint for the $\widehat{g}_{2 n}$}

We can propose an Ansatz for the perturbative corrections to $\widehat{g}_{2 n}$ correlators similar to (4.2). It reads

$$
\begin{aligned}
\widehat{g}_{2 n}= & \frac{c_{N} n ! 2^{2 n}}{(\operatorname{Im} \tau)^{2 n+3}} \frac{\Gamma\left(\frac{N^{2}-1}{2}+3+n\right)}{\Gamma\left(\frac{N^{2}-1}{2}+3\right)}\left[1+\mathcal{B}_{2} \frac{n^{2}+\eta_{2,1} n+\eta_{2,0}}{(\operatorname{Im} \tau)^{2}}+\mathcal{B}_{3} \frac{n^{3}+\eta_{3,2} n^{2}+\eta_{3,1} n+\eta_{3,0}}{(\operatorname{Im} \tau)^{3}}\right. \\
& \left.+\mathcal{B}_{4} \frac{n^{4}+\eta_{4,3} n^{3}+\eta_{4,2} n^{2}+\eta_{4,1} n+\eta_{4,0}}{(\operatorname{Im} \tau)^{4}}+\ldots\right]
\end{aligned}
$$

where $c_{N}=\frac{3\left(N^{2}-4\right)\left(N^{2}-1\right)}{N}$. Compared with (4.2), we see that in this case the corrections do not vanish for $n=0$ because $\widehat{g}_{0}=\left\langle\operatorname{Tr} \varphi^{3} \operatorname{Tr} \bar{\varphi}^{3}\right\rangle$ is clearly non-trivial. Again, imposing the Toda equation, we can fix all the $\eta$-coefficients but not the $\mathcal{B}_{k}$ ones. For instance, one finds at first order

$$
\eta_{2,1}=\frac{1}{2}\left(N^{2}+5\right), \quad \eta_{2,0}=\frac{1}{48}\left[2\left(35+12 N^{2}+N^{4}\right)+\frac{9\left(N^{4}-1\right) \zeta(3)}{\pi^{2} \mathcal{B}_{2}}\right] .
$$

Matching the Ansatz (5.5) to the $n=0$ case gives then

$$
\mathcal{B}_{2}=-\frac{9 \zeta(3)}{2 \pi^{2}}
$$

We carried on this procedure up to $\mathcal{O}\left(g^{14}\right)$. The ratio $\mathrm{SQCD} / \mathcal{N}=4$ is now expressed by

$$
\widehat{F}(g, n ; N)=\frac{\left.\widehat{g}_{2 n}\right|_{\mathrm{SQCD}}}{\left.\widehat{g}_{2 n}\right|_{\mathcal{N}=4}}=1+\sum_{k=2}^{\infty} \frac{\widehat{f}_{k}(n ; N)}{(\operatorname{Im} \tau)^{k}},
$$

where

$$
\begin{aligned}
f_{2}(n ; N)= & -\frac{9(n+1)\left(N^{2}+2 n+3\right) \zeta(3)}{4 \pi^{2}} \\
f_{3}(n ; N)= & \frac{5\left(N^{2}-1\right)}{16 \pi^{3} N\left(N^{2}+5\right)\left(N^{2}+7\right)\left(N^{2}+9\right)}\left[105(3+2 n)\left(53+90 n+40 n^{2}\right)\right. \\
& +\left(14509+30780 n+19800 n^{2}+3600 n^{3}\right) N^{2}+\left(4259+7952 n+3300 n^{2}+160 n^{3}\right) N^{4} \\
& \left.+\left(515+804 n+120 n^{2}\right) N^{6}+2(11+12 n) N^{8}\right] \zeta(5),
\end{aligned}
$$




$$
\begin{aligned}
f_{4}(n ; N)= & \frac{9}{32 \pi^{4}}\left[(3+2 n)\left(74+153 n+99 n^{2}+18 n^{3}\right)+12(1+n)\left(10+12 n+3 n^{2}\right) N^{2}\right. \\
& \left.+\left(18+29 n+9 n^{2}\right) N^{4}\right] \zeta(3)^{2}-\frac{35}{64 \pi^{4} N^{2}\left(N^{2}+5\right)\left(N^{2}+7\right)\left(N^{2}+9\right)\left(N^{2}+11\right)} \\
& {\left[3465(1+n)(3+2 n)\left(29+35 n+14 n^{2}\right)\right.} \\
& -3\left(688+24675 n+62370 n^{2}+55860 n^{3}+17640 n^{4}\right) N^{2} \\
& +2\left(33327+98135 n+118060 n^{2}+71890 n^{3}+19670 n^{4}\right) N^{4} \\
& +5\left(33429+91204 n+95074 n^{2}+44268 n^{3}+7280 n^{4}\right) N^{6} \\
& +\left(74319+182900 n+148445 n^{2}+42000 n^{3}+1120 n^{4}\right) N^{8} \\
& +\left(13446+29405 n+15540 n^{2}+1120 n^{3}\right) N^{10} \\
& \left.+4\left(273+505 n+90 n^{2}\right) N^{12}+(33+40 n) N^{14}\right] \zeta(7),
\end{aligned}
$$

and so on. ${ }^{14}$

\subsection{Large R-charge limit}

In the large R-charge limit (1.2) we have the following expansion of the logarithm of the function $\widehat{F}(\lambda, N)=\lim _{n \rightarrow \infty} \widehat{F}(\sqrt{\lambda / n}, n ; N)$

$$
\begin{aligned}
& \log \widehat{F}(\lambda ; N)=-\frac{9 \lambda^{2} \boldsymbol{\zeta}(\mathbf{3})}{32 \pi^{4}}+\frac{25 \lambda^{3}\left(N^{2}-1\right)\left(2 N^{4}+45 N^{2}+105\right) \boldsymbol{\zeta}(\mathbf{5})}{64 \pi^{6} N\left(N^{2}+5\right)\left(N^{2}+7\right)\left(N^{2}+9\right)} \\
& -\frac{1225 \lambda^{4}\left(8 N^{8}+260 N^{6}+281 N^{4}-378 N^{2}+693\right) \boldsymbol{\zeta}(\mathbf{7})}{4096 \pi^{8} N^{2}\left(N^{2}+5\right)\left(N^{2}+7\right)\left(N^{2}+9\right)\left(N^{2}+11\right)} \\
& +\frac{1323 \lambda^{5}\left(N^{2}-1\right)\left(26 N^{8}+1180 N^{6}+3309 N^{4}+3096 N^{2}+3861\right) \boldsymbol{\zeta}(\mathbf{9})}{4096 \pi^{10} N^{3}\left(N^{2}+5\right)\left(N^{2}+7\right)\left(N^{2}+9\right)\left(N^{2}+11\right)\left(N^{2}+13\right)} \\
& +\lambda^{6}\left(\frac{5775}{8192 \pi^{12}\left(N^{2}+5\right)^{2}\left(N^{2}+7\right)^{2}\left(N^{2}+9\right)^{2}\left(N^{2}+11\right)\left(N^{2}+13\right)\left(N^{2}+15\right)}\right. \\
& \quad\left(N^{14}+88 N^{12}+15 N^{10}-18088 N^{8}-39661 N^{6}+1053540 N^{4}+4281405 N^{2}+4399500\right) \boldsymbol{\zeta}(\mathbf{5})^{2} \\
& \left.-\frac{17787\left(122 N^{12}+6950 N^{10}+24848 N^{8}+8085 N^{6}-12645 N^{4}+15345 N^{2}+32175\right) \boldsymbol{\zeta}(\mathbf{1 1})}{65536 \pi^{12} N^{4}\left(N^{2}+5\right)\left(N^{2}+7\right)\left(N^{2}+9\right)\left(N^{2}+11\right)\left(N^{2}+13\right)\left(N^{2}+15\right)}\right) \\
& +\lambda^{7}\left(\frac{552123\left(N^{2}-1\right)}{131072 \pi^{14} N^{5}\left(N^{2}+5\right)\left(N^{2}+7\right)\left(N^{2}+9\right)\left(N^{2}+11\right)\left(N^{2}+13\right)\left(N^{2}+15\right)\left(N^{2}+17\right)}\right. \\
& \left(34 N^{12}+2424 N^{10}+17285 N^{8}+29655 N^{6}+24450 N^{4}+41145 N^{2}+16575\right) \boldsymbol{\zeta}(\mathbf{1 3}) \\
& -\frac{15015\left(N^{2}-1\right)}{32768 \pi^{14} N\left(N^{2}+5\right)^{2}\left(N^{2}+7\right)^{2}\left(N^{2}+9\right)^{2}\left(N^{2}+11\right)\left(N^{2}+13\right)\left(N^{2}+15\right)\left(N^{2}+17\right)} \\
& \left(22 N^{14}+2323 N^{12}+6951 N^{10}-473938 N^{8}-1641088 N^{6}+30589515 N^{4}\right. \\
& \left.\left.+136118115 N^{2}+144364500\right) \boldsymbol{\zeta}(\mathbf{5}) \boldsymbol{\zeta}(\mathbf{7})\right)+\mathcal{O}\left(\lambda^{8}\right),
\end{aligned}
$$

where we have emphasized the $\zeta$-functions. Compared to $F(\lambda ; N)$, the $\zeta(3)$-dependent terms again exponentiate and are independent on $N$. Apart from this, there are no further

\footnotetext{
${ }^{14}$ Again, the next functions are available under request.
} 
special simplifications for low values of $N$. The first non-trivial specialized cases are $N=$ 3,4 that give ${ }^{15}$

$$
\begin{aligned}
\log \widehat{F}(\lambda ; 3)= & -\frac{9 \lambda^{2} \zeta(3)}{32 \pi^{4}}+\frac{25 \lambda^{3} \zeta(5)}{144 \pi^{6}}-\frac{15925 \lambda^{4} \zeta(7)}{147456 \pi^{8}}+\frac{147 \lambda^{5} \zeta(9)}{2048 \pi^{10}} \\
& +\lambda^{6}\left(\frac{1925 \zeta(5)^{2}}{14155776 \pi^{12}}-\frac{8599591 \zeta(11)}{169869312 \pi^{12}}\right)+\lambda^{7}\left(\frac{3177031 \zeta(13)}{84934656 \pi^{14}}-\frac{5005 \zeta(5) \zeta(7)}{14155776 \pi^{14}}\right)+\mathcal{O}\left(\lambda^{8}\right), \\
\log \widehat{F}(\lambda ; 4)= & -\frac{9 \lambda^{2} \zeta(3)}{32 \pi^{4}}+\frac{955 \lambda^{3} \zeta(5)}{5888 \pi^{6}}-\frac{429289 \lambda^{4} \zeta(7)}{4521984 \pi^{8}}+\frac{78057 \lambda^{5} \zeta(9)}{1310720 \pi^{10}} \\
& +\lambda^{6}\left(\frac{146531 \zeta(5)^{2}}{973969408 \pi^{12}}-\frac{68971014343 \zeta(11)}{1734512476160 \pi^{12}}\right) \\
& +\lambda^{7}\left(\frac{387146868537 \zeta(13)}{13876099809280 \pi^{14}}-\frac{28131103 \zeta(5) \zeta(7)}{77917552640 \pi^{14}}\right)+\mathcal{O}\left(\lambda^{8}\right) .
\end{aligned}
$$

\section{Relation with large $N$ factorization}

Given our results that hold for all values of the R-charge $n$ and gauge rank $N$, it is interesting to consider their large $N$ expansion, see also [58-60]. To this aim, let us define in this section the conventional 't Hooft coupling $\lambda=N g^{2}$, not to be confused with the large R-charge coupling in (1.2). We are interested in

$$
\mathcal{F}_{n}(\lambda)=\lim _{N \rightarrow \infty} F(\sqrt{\lambda / N}, n ; N)
$$

where $F(g, n ; N)$ is in $(2.15) .{ }^{16}$ Using the results in (4.6), we find

$$
\log \mathcal{F}_{n}(\lambda)=n \log \mathcal{F}_{1}(\lambda)
$$

with (we write only the first 7 orders, but we checked (6.2) at order $\mathcal{O}\left(\lambda^{10}\right)$ )

$$
\begin{aligned}
\log \mathcal{F}_{1}(\lambda)= & -\frac{9 \lambda^{2} \zeta(3)}{64 \pi^{4}}+\frac{15 \lambda^{3} \zeta(5)}{128 \pi^{6}}+\lambda^{4}\left(\frac{99 \zeta(3)^{2}}{8192 \pi^{8}}-\frac{175 \zeta(7)}{2048 \pi^{8}}\right)+\lambda^{5}\left(\frac{4095 \zeta(9)}{65536 \pi^{10}}-\frac{405 \zeta(3) \zeta(5)}{16384 \pi^{10}}\right) \\
& +\lambda^{6}\left(-\frac{189 \zeta(3)^{3}}{131072 \pi^{12}}+\frac{6375 \zeta(5)^{2}}{524288 \pi^{12}}+\frac{2835 \zeta(3) \zeta(7)}{131072 \pi^{12}}-\frac{98637 \zeta(11)}{2097152 \pi^{12}}\right) \\
& +\lambda^{7}\left(\frac{495 \zeta(3)^{2} \zeta(5)}{1048576 \pi^{14}}-\frac{21595 \zeta(5) \zeta(7)}{1048576 \pi^{14}}-\frac{77805 \zeta(3) \zeta(9)}{4194304 \pi^{14}}+\frac{153153 \zeta(13)}{4194304 \pi^{14}}\right) \\
& +\lambda^{8}\left(\frac{13203 \zeta(3)^{4}}{67108864 \pi^{16}}-\frac{169425 \zeta(3) \zeta(5)^{2}}{33554432 \pi^{16}}-\frac{38115 \zeta(3)^{2} \zeta(7)}{8388608 \pi^{16}}+\frac{568645 \zeta(7)^{2}}{67108864 \pi^{16}}\right. \\
& \left.+\frac{144585 \zeta(5) \zeta(9)}{8388608 \pi^{16}}+\frac{2155923 \zeta(3) \zeta(11)}{134217728 \pi^{16}}-\frac{7818525 \zeta(15)}{268435456 \pi^{16}}\right)+\ldots
\end{aligned}
$$

\footnotetext{
${ }^{15}$ Here it does not make sense to set $N=2$ since the correlators $\widehat{g}_{2 n}$ are zero due to $\operatorname{Tr} \varphi^{3}=0$ in $\mathrm{SU}(2)$. This is consistent with $c_{2}=0$ in (5.5).

${ }^{16}$ It is important to remark that absence of instanton corrections at large $N$ is based on the assumption that the instanton moduli integration does not spoil the instanton action exponential suppression as $g \rightarrow 0$. In general, this may be a non-trivial issues, and instantons may lead to large $N$ phase transitions, see for instance [61]. A test of this assumption in $\mathcal{N}=2$ SQCD is discussed in [21] where it is shown that the one-instanton corrections to the partition function leads to a $\sqrt{N}$ enhancement of the exponentially small instanton action factor. Thus, it is negligible at large $N$.
} 
Relation (6.2) expresses factorization

$$
\left\langle\left(\operatorname{Tr} \varphi^{2}\right)^{n}\left(\operatorname{Tr} \bar{\varphi}^{2}\right)^{n}\right\rangle \sim\left\langle\operatorname{Tr} \varphi^{2} \operatorname{Tr} \bar{\varphi}^{2}\right\rangle^{n}
$$

at leading large $N$. The finite $N$ corrections to $(6.2)$ are $\mathcal{O}\left(\frac{n}{N^{2}}, \frac{n^{2}}{N^{2}}\right)$, where we explicitly denoted that the dependence on $n$ is the sum of linear and quadratic pieces.

A similar analysis for the function $\widehat{\mathcal{F}}$ which is the large $N$ limit of the function $\widehat{F}$ in (5.8) shows that (6.2) reads now

$$
\log \frac{\widehat{\mathcal{F}}_{n}(\lambda)}{\widehat{\mathcal{F}}_{0}(\lambda)}=n \log \mathcal{F}_{1}(\lambda)
$$

where $\log \mathcal{F}_{1}(\lambda)$ is the expression in (6.3). Again, this implies the following large $N$ factorization

$$
\left\langle\left(\operatorname{Tr} \varphi^{2}\right)^{n} \operatorname{Tr} \varphi^{3}\left(\operatorname{Tr} \bar{\varphi}^{2}\right)^{n} \operatorname{Tr} \bar{\varphi}^{3}\right\rangle \sim\left\langle\operatorname{Tr} \varphi^{2} \operatorname{Tr} \bar{\varphi}^{2}\right\rangle^{n}\left\langle\operatorname{Tr} \varphi^{3} \operatorname{Tr} \bar{\varphi}^{3}\right\rangle
$$

Subleading corrections to large $N$ may of course be extracted easily from the general expressions valid for generic $N$.

\section{Acknowledgments}

We thank A. Bourget, D. Rodriguez-Gomez, and Jorge G. Russo for discussions and kind clarifications about their work. We also thank A. A. Tseytlin for useful suggestions and comments.

\section{A Functions $F^{(\ell)}(N)$ for $\ell=8,9,10$}

Let us define

$$
\mathrm{P}_{k}=\left(\frac{N^{2}+1}{2}\right)_{k}, \quad(x)_{k}=\frac{\Gamma(x+k)}{\Gamma(x)} .
$$

The functions $F^{(\ell)}(N)$ defined in section 4 for $\ell=8,9,10$ are given by the following expressions.

$$
\begin{aligned}
F_{7^{2}}^{(8)}(N)= & \frac{1576575\left(N^{2}-4\right)}{256 N^{2} \mathrm{P}_{3} \mathrm{P}_{7}}\left(98 N^{14}+928 N^{12}-4151 N^{10}-44359 N^{8}-42036 N^{6}\right. \\
& \left.+26754 N^{4}+14553 N^{2}-16299\right), \\
F_{5,9}^{(8)}(N)= & \frac{675675\left(N^{2}-4\right)}{2 N^{2}\left(N^{2}+3\right) \mathrm{P}_{7}}\left(23 N^{10}+70 N^{8}-1455 N^{6}-1335 N^{4}+192 N^{2}-855\right) \\
F_{15}^{(8)}(N)= & -\frac{41409225}{256 N^{6} \mathrm{P}_{7}}\left(540 N^{14}+3780 N^{12}+4676 N^{10}+440 N^{8}+329 N^{6}\right. \\
& \left.-735 N^{4}+735 N^{2}+315\right), \\
F_{5^{3}}^{(9)}(N)= & \frac{3038750\left(N^{2}-4\right)}{3 N\left(N^{2}+3\right)^{2} \mathrm{P} 8}\left(2 N^{12}-28 N^{10}-511 N^{8}+2187 N^{6}+24479 N^{4}+8701 N^{2}-7950\right),
\end{aligned}
$$




$$
\begin{aligned}
F_{7,9}^{(9)}(N)= & -\frac{1276275\left(N^{2}-4\right)}{32 N^{3} \mathrm{P}_{3} \mathrm{P}_{8}}\left(724 N^{16}+9884 N^{14}-19378 N^{12}-455219 N^{10}\right. \\
& \left.-693063 N^{8}-78342 N^{6}+24156 N^{4}-48195 N^{2}+162729\right), \\
F_{5,11}^{(9)}(N)=- & \frac{10027875\left(N^{2}-4\right)}{8 N^{3}\left(N^{2}+3\right) \mathrm{P}_{8}}\left(142 N^{12}+926 N^{10}-9807 N^{8}\right. \\
& \left.-23606 N^{6}-3754 N^{4}+2184 N^{2}+7035\right), \\
F_{17}^{(9)}(N)= & \frac{29548805}{32 N^{7} \mathrm{P}_{8}}\left(1866 N^{16}+19976 N^{14}+45374 N^{12}\right. \\
& \left.+17804 N^{10}+345 N^{8}-1260 N^{6}+4410 N^{4}-6300 N^{2}-1575\right), \\
F_{5^{2}, 7}^{(10)}(N)= & -\frac{121246125\left(N^{2}-4\right)\left(N^{2}-1\right)}{32 N^{2}\left(N^{2}+3\right) \mathrm{P}_{3} \mathrm{P}_{9}}\left(7 N^{16}-16 N^{14}-2593 N^{12}-11454 N^{10}\right. \\
& \left.+132421 N^{8}+888024 N^{6}+1182001 N^{4}+282422 N^{2}-148380\right), \\
F_{92}^{(10)}(N)= & \frac{2909907\left(N^{2}-4\right)}{64 N^{4}\left(N^{2}+7\right) \mathrm{P}_{3} \mathrm{P}_{9}}\left(7697 N^{20}+205301 N^{18}+1156794 N^{16}\right. \\
& -6284951 N^{14}-65595428 N^{12}-119443263 N^{10}-65064156 N^{8} \\
& \left.-37063809 N^{6}-36848169 N^{4}-7387038 N^{2}-18683298\right), \\
F_{7,11}^{(10)}(N)= & \frac{53348295\left(N^{2}-4\right)}{256 N^{4} \mathrm{P}_{3} \mathrm{P}_{9}}\left(3218 N^{18}+60754 N^{16}+34194 N^{14}-2724467 N^{12}\right. \\
& \left.-7509568 N^{10}-3899769 N^{8}+2216346 N^{6}+1637547 N^{4}-330750 N^{2}-1099665\right), \\
F_{5,13}^{(10)}(N)= & \frac{297226215\left(N^{2}-4\right)}{8 N^{4}\left(N^{2}+3\right) \mathrm{P}_{9}}\left(111 N^{14}+1214 N^{12}-6968 N^{10}-42034 N^{8}-20548 N^{6}\right. \\
& \left.+5225 N^{4}-9555 N^{2}-8085\right), \\
F_{19}^{(10)}(N)= & -\frac{2133423721}{640 N^{8} \mathrm{P}_{9}}\left(10688 N^{18}+165378 N^{16}+616452 N^{14}+541822 N^{12}\right. \\
& \left.+49410 N^{10}+8235 N^{8}-11340 N^{6}-28350 N^{4}+85050 N^{2}+14175\right) .
\end{aligned}
$$

\section{B The correlators $\widehat{g}_{2 n}$ for $n=2,3$}

The explicit expressions for the correlators $\widehat{g}_{2 n}$ for $n=2,3$ are

$$
\begin{aligned}
\widehat{g}_{4}= & \frac{24\left(N^{2}-1\right)\left(N^{2}-4\right)\left(N^{2}+5\right)\left(N^{2}+7\right)}{N}\left(\frac{g^{2}}{4 \pi}\right)^{7}\left[1-\frac{27 g^{4}\left(N^{2}+7\right) \zeta(3)}{64 \pi^{4}}\right. \\
& +\frac{5 g^{6}\left(N^{2}-1\right)\left(70 N^{6}+1973 N^{4}+16886 N^{2}+32095\right) \zeta(5)}{1024 \pi^{6} N\left(N^{2}+5\right)\left(N^{2}+7\right)} \\
& +g^{8}\left(\frac{9\left(14 N^{4}+207 N^{2}+805\right) \zeta(3)^{2}}{1024 \pi^{8}}\right. \\
& \left.-\frac{35\left(113 N^{10}+4312 N^{8}+45949 N^{6}+41951 N^{4}-54186 N^{2}+113925\right) \zeta(7)}{16384 \pi^{8} N^{2}\left(N^{2}+5\right)\left(N^{2}+7\right)}\right) \\
& +g^{10}\left(\frac{63\left(N^{2}-1\right)\left(712 N^{10}+37376 N^{8}+575639 N^{6}+1477845 N^{4}+1403305 N^{2}+1644195\right) \zeta(9)}{262144 \pi^{10} N^{3}\left(N^{2}+5\right)\left(N^{2}+7\right)}\right. \\
& \left.\left.-\frac{45\left(N^{2}-1\right)\left(310 N^{8}+11471 N^{6}+154469 N^{4}+833209 N^{2}+1319325\right) \zeta(3) \zeta(5)}{65536 \pi^{10} N\left(N^{2}+5\right)\left(N^{2}+7\right)}\right)+\mathcal{O}\left(g^{12}\right)\right],
\end{aligned}
$$




$$
\begin{aligned}
\widehat{g}_{6}= & \frac{144\left(N^{2}-1\right)\left(N^{2}-4\right)\left(N^{2}+5\right)\left(N^{2}+7\right)\left(N^{2}+9\right)}{N}\left(\frac{g^{2}}{4 \pi}\right)^{9}\left[1-\frac{9 g^{4}\left(N^{2}+9\right) \zeta(3)}{16 \pi^{4}}\right. \\
& +\frac{5 g^{6}\left(N^{2}-1\right)\left(94 N^{8}+4007 N^{6}+62135 N^{4}+382249 N^{2}+645435\right) \zeta(5)}{1024 \pi^{6} N\left(N^{2}+5\right)\left(N^{2}+7\right)\left(N^{2}+9\right)} \\
& +g^{8}\left(\frac{27\left(31 N^{4}+584 N^{2}+2865\right) \zeta(3)^{2}}{4096 \pi^{8}}\right. \\
& \left.-\frac{105\left(51 N^{12}+2903 N^{10}+58654 N^{8}+416054 N^{6}+336111 N^{4}-414173 N^{2}+982800\right) \zeta(7)}{16384 \pi^{8} N^{2}\left(N^{2}+5\right)\left(N^{2}+7\right)\left(N^{2}+9\right)}\right) \\
& +g^{10}\left(\frac { 6 3 ( N ^ { 2 } - 1 ) } { 2 6 2 1 4 4 \pi ^ { 1 0 } N ^ { 3 } ( N ^ { 2 } + 5 ) ( N ^ { 2 } + 7 ) ( N ^ { 2 } + 9 ) } \left(972 N^{12}+74304 N^{10}+2058613 N^{8}\right.\right. \\
& \left.+21049036 N^{6}+50557990 N^{4}+48478260 N^{2}+53986905\right) \zeta(9) \\
& \left.-\frac{45\left(N^{2}-1\right)\left(128 N^{10}+6850 N^{8}+145489 N^{6}+1476329 N^{4}+6789279 N^{2}+9999045\right) \zeta(3) \zeta(5)}{16384 \pi^{10} N\left(N^{2}+5\right)\left(N^{2}+7\right)\left(N^{2}+9\right)}\right) \\
& \left.+\mathcal{O}\left(g^{12}\right)\right] .
\end{aligned}
$$

\section{Decoupling violations and Toda equation for general towers}

Let us clarify to what extent we may have decoupling and reduction to a single semi-infinite Toda equation for more general towers of the form $\Phi^{(n)}=\left(\operatorname{Tr} \varphi^{2}\right)^{n} \Phi$ with $\operatorname{dim} \Phi>3$. Although general discussions already appeared in [25, 26], we consider here in full details the example of $\Phi=\operatorname{Tr} \varphi^{4}$ and consider in somewhat more details what is the fate of the would-be decoupled Toda equation.

Compared with the case $\Phi=\operatorname{Tr} \varphi^{3}$, an important difference is that Wick contraction inside $\operatorname{Tr} \varphi^{4}$ brings back to the tower of the identity $\Phi=\mathbb{I}$. Let us work at the level of matrix model traces $t_{\boldsymbol{n}}$, cf. (3.5) and denote by : $T$ :, the operator $T$ with resolved mixing, i.e. after subtraction of the suitable contraction ordering contributions, i.e. as in the r.h.s. of (3.11). ${ }^{17}$ At dimension $4+2 n$, we can consider the two operators

$$
\mathcal{O}_{n}=: \underbrace{t_{2, \ldots, 2,4}}_{n}:, \quad \mathcal{O}_{n}^{\prime}=: \underbrace{t_{2, \ldots, 2}}_{n+2}: .
$$

These are natural in the sense that they map to the simple flat space operators

$$
\mathcal{O}_{n}^{\mathbb{R}^{4}}=\left(\operatorname{Tr} \varphi^{2}\right)^{n} \operatorname{Tr} \varphi^{4}, \quad \mathcal{O}_{n}^{\prime} \mathbb{R}^{4}=\left(\operatorname{Tr} \varphi^{2}\right)^{n+2} .
$$

Besides, we can construct the operator

$$
\widetilde{\mathcal{O}}_{n}=\mathcal{O}_{n}-\kappa_{n}(g) \mathcal{O}_{n}^{\prime}
$$

where $\kappa_{n}(g)$ is chosen such that $\left\langle\widetilde{\mathcal{O}}_{n} \overline{\mathcal{O}}_{n}^{\prime}\right\rangle=0$. The second term in (C.3) is a further subtraction unrelated to $S^{4}$ mixing, since the two operators have the same dimension. Instead, it is a loop corrected orthogonalization similar in spirit to the tree level diagonalization discussed in [25], and shown to be problematic due to three loop corrections in [26].

\footnotetext{
${ }^{17}$ Notice that in the notation of [45], this is denoted $: X: g$ to emphasize the difference with respect to the $\mathcal{N}=4$ limit.
} 
Now, it should be clearly stated that the 2-point function of $\widetilde{\mathcal{O}}_{n}$ do satisfy the decoupled Toda equation (2.9), contrary to $\mathcal{O}_{n}$. So, decoupling is possible, but the point is that it is not achieved in a natural way because the coefficients $\kappa_{n}(g)$ in (C.3) depend on $n$. This means that, for instance, the large $n$ limit has to take into account the extra $n$ dependence in $\kappa_{n}(g)$.

To see this in details, let us work out the explicit expressions of the above operators at level $n=0$, i.e. dimension 4 . We find

$$
\begin{aligned}
\mathcal{O}_{0}= & t_{4}-\frac{\left(2 N^{2}-3\right)}{N\left(N^{2}+1\right)} t_{2,2} \\
& -\frac{25\left(N^{2}-9\right)\left(N^{2}-4\right) \zeta(5)}{256 \pi^{6}\left(N^{2}+1\right)}\left[t_{2,2}+\frac{3}{20}\left(N^{4}-1\right)-\frac{3}{4}\left(N^{2}+1\right) t_{2}\right] g^{6}+\mathcal{O}\left(g^{8}\right), \quad(\text { C. } 4) \\
\mathcal{O}_{0}^{\prime}= & t_{2,2}+\frac{1}{4}\left(N^{4}-1\right)-\left(N^{2}+1\right) t_{2} \frac{3\left(N^{2}+1\right)\left(N^{2}+5\right) \zeta(3)}{64 \pi^{4}}\left[t_{2}-\frac{\left(N^{2}-1\right)\left(N^{2}+4\right)}{2\left(N^{2}+5\right)}\right] g^{4} \\
& -\frac{15\left(N^{2}+1\right)\left(N^{2}+11\right)\left(2 N^{2}-1\right) \zeta(5)}{1024 \pi^{6} N}\left[t_{2}-\frac{\left(N^{2}-1\right)\left(N^{2}+9\right)}{2\left(N^{2}+11\right)}\right] g^{6}+\mathcal{O}\left(g^{8}\right) . \quad(\text { C. } 5)
\end{aligned}
$$

The condition $\left\langle\widetilde{\mathcal{O}}_{0} \overline{\mathcal{O}}_{0}^{\prime}\right\rangle=\left\langle\left[\mathcal{O}_{0}-\kappa_{0}(g) \mathcal{O}_{0}^{\prime}\right] \overline{\mathcal{O}}_{0}^{\prime}\right\rangle=0$ gives

$$
\kappa_{0}(g)=\frac{2 N^{2}-3}{N\left(N^{2}+1\right)}+\frac{25\left(N^{2}-9\right)\left(N^{2}-4\right) \zeta(5)}{256 \pi^{6}\left(N^{2}+1\right)} g^{6}+\mathcal{O}\left(g^{8}\right) .
$$

Repeating the calculation for $n=1,2,3$ gives a similar result but with a modified $\mathcal{O}\left(g^{6}\right)$ term

$$
\kappa_{n}(g)=\frac{2 N^{2}-3}{N\left(N^{2}+1\right)}+\frac{25\left(N^{2}-9\right)\left(N^{2}-4\right) \zeta(5)}{256 \pi^{6}\left(N^{2}+1\right)} \alpha_{n} g^{6}+\mathcal{O}\left(g^{8}\right),
$$

where

$$
\alpha_{1}=\frac{N^{2}+11}{N^{2}+3}, \quad \alpha_{2}=\frac{\left(N^{2}+9\right)\left(N^{2}+15\right)}{\left(N^{2}+3\right)\left(N^{2}+5\right)}, \quad \alpha_{3}=\frac{\left(N^{2}+9\right)\left(N^{2}+11\right)\left(N^{2}+19\right)}{\left(N^{2}+3\right)\left(N^{2}+5\right)\left(N^{2}+7\right)} .
$$

As anticipated, the coefficient $\kappa_{n}(g)$ depends on $n$ starting at three loops.

Let us now show that the 2-point function $\widetilde{g}_{2 n}$ of the $\widetilde{\mathcal{O}}_{n}$ operators is still captured by the Toda equation. We find (the tree level is known, see for instance [26])

$$
\widetilde{g}_{2 n}=\frac{4\left(N^{2}-1\right)\left(N^{2}-4\right)\left(N^{2}-9\right)}{N^{2}+1} \frac{n ! 2^{2 n}}{(\operatorname{Im} \tau)^{2 n+4}} \frac{\Gamma\left(\frac{N^{2}-1}{2}+4+n\right)}{\Gamma\left(\frac{N^{2}-1}{2}+4\right)}\left(1+\delta \widetilde{g}_{2 n}\right),
$$

with

$$
\begin{aligned}
\delta \widetilde{g}_{0}= & -\frac{3 g^{4}\left(N^{2}+4\right) \zeta(3)}{16 \pi^{4}}+\frac{5 g^{6}\left(N^{2}-1\right)\left(8 N^{2}+3\right) \zeta(5)}{256 \pi^{6} N}+\mathcal{O}\left(g^{8}\right) \\
\delta \widetilde{g}_{2}= & -\frac{3 g^{4}\left(7 N^{2}+43\right) \zeta(3)}{64 \pi^{4}}+\frac{5 g^{6}\left(N^{2}-1\right)\left(7 N^{4}+127 N^{2}+45\right) \zeta(5)}{128 \pi^{6} N\left(N^{2}+7\right)}+\mathcal{O}\left(g^{8}\right) \\
\delta \widetilde{g}_{4}= & -\frac{3 g^{4}\left(5 N^{2}+41\right) \zeta(3)}{32 \pi^{4}}+\frac{5 g^{6}\left(N^{2}-1\right)\left(20 N^{6}+689 N^{4}+6150 N^{2}+2121\right) \zeta(5)}{256 \pi^{6} N\left(N^{2}+7\right)\left(N^{2}+9\right)}+\mathcal{O}\left(g^{8}\right) \\
\delta \widetilde{g}_{6}= & -\frac{3 g^{4}\left(13 N^{2}+133\right) \zeta(3)}{64 \pi^{4}} \\
& +\frac{5 g^{6}\left(N^{2}-1\right)\left(13 N^{8}+672 N^{6}+11876 N^{4}+71268 N^{2}+24051\right) \zeta(5)}{128 \pi^{6} N\left(N^{2}+7\right)\left(N^{2}+9\right)\left(N^{2}+11\right)}+\mathcal{O}\left(g^{8}\right)
\end{aligned}
$$


Using (C.9) and (C.10), one can check that the first instances of the Toda equation (2.9) are satisfied. This includes the effect of the 3-loop $\zeta(5)$ terms which are responsible for the $g$-dependent mixing discussed in [26]. This means that it is easy to obtain closed expressions for the expansions in (C.10) by the methods discussed in the main text. For instance, the first correction in (C.10) is

$$
\delta \widetilde{g}_{2 n}=-\frac{3\left[16+21 n+6 n^{2}+(4+3 n) N^{2}\right]}{64 \pi^{4}} \zeta(3) g^{4}+\ldots,
$$

while higher order corrections may be treated as we discussed the $\mathbb{I}$ and $\operatorname{Tr} \varphi^{3}$ towers.

Open Access. This article is distributed under the terms of the Creative Commons Attribution License (CC-BY 4.0), which permits any use, distribution and reproduction in any medium, provided the original author(s) and source are credited.

\section{References}

[1] K.A. Intriligator, Bonus symmetries of $N=4$ superYang-Mills correlation functions via AdS duality, Nucl. Phys. B 551 (1999) 575 [hep-th/9811047] [INSPIRE].

[2] E. D'Hoker, D.Z. Freedman and W. Skiba, Field theory tests for correlators in the AdS/CFT correspondence, Phys. Rev. D 59 (1999) 045008 [hep-th/9807098] [INSPIRE].

[3] S. Lee, S. Minwalla, M. Rangamani and N. Seiberg, Three point functions of chiral operators in $D=4, N=4 S Y M$ at large $N$, Adv. Theor. Math. Phys. 2 (1998) 697 [hep-th/9806074] [INSPIRE].

[4] E. D'Hoker, D.Z. Freedman, S.D. Mathur, A. Matusis and L. Rastelli, Extremal correlators in the AdS/CFT correspondence, hep-th/9908160 [INSPIRE].

[5] M. Baggio, J. de Boer and K. Papadodimas, A non-renormalization theorem for chiral primary 3-point functions, JHEP 07 (2012) 137 [arXiv:1203.1036] [INSPIRE].

[6] N. Beisert et al., Review of AdS/CFT Integrability: An Overview, Lett. Math. Phys. 99 (2012) 3 [arXiv: 1012.3982] [INSPIRE].

[7] N. Gromov, V. Kazakov, S. Leurent and D. Volin, Quantum Spectral Curve for Planar $\mathcal{N}=4$ Super-Yang-Mills Theory, Phys. Rev. Lett. 112 (2014) 011602 [arXiv: 1305.1939] [INSPIRE].

[8] N. Gromov, Introduction to the Spectrum of $N=4$ SYM and the Quantum Spectral Curve, arXiv: 1708.03648 [INSPIRE].

[9] J.M. Maldacena, Wilson loops in large N field theories, Phys. Rev. Lett. 80 (1998) 4859 [hep-th/9803002] [INSPIRE].

[10] V. Pestun et al., Localization techniques in quantum field theories, J. Phys. A 50 (2017) 440301 [arXiv: 1608.02952] [INSPIRE].

[11] J.K. Erickson, G.W. Semenoff and K. Zarembo, Wilson loops in $N=4$ supersymmetric Yang-Mills theory, Nucl. Phys. B 582 (2000) 155 [hep-th/0003055] [INSPIRE].

[12] N. Drukker and D.J. Gross, An exact prediction of $N=4$ SUSYM theory for string theory, J. Math. Phys. 42 (2001) 2896 [hep-th/0010274] [INSPIRE].

[13] V. Pestun, Localization of gauge theory on a four-sphere and supersymmetric Wilson loops, Commun. Math. Phys. 313 (2012) 71 [arXiv:0712.2824] [InSPIRE]. 
[14] D. Medina-Rincon, A.A. Tseytlin and K. Zarembo, Precision matching of circular Wilson loops and strings in $A d S_{5} \times S^{5}$, JHEP 05 (2018) 199 [arXiv:1804.08925] [INSPIRE].

[15] N. Seiberg and E. Witten, Electric-magnetic duality, monopole condensation and confinement in $N=2$ supersymmetric Yang-Mills theory, Nucl. Phys. B 426 (1994) 19 [Erratum ibid. B 430 (1994) 485] [hep-th/9407087] [INSPIRE].

[16] N. Seiberg and E. Witten, Monopoles, duality and chiral symmetry breaking in $N=2$ supersymmetric QCD, Nucl. Phys. B 431 (1994) 484 [hep-th/9408099] [INSPIRE].

[17] J. Teschner, Exact Results on $\mathcal{N}=2$ Supersymmetric Gauge Theories, in New Dualities of Supersymmetric Gauge Theories, J. Teschner ed., Springer, (2015), pp. 1-30, arXiv: 1412.7145 .

[18] N.A. Nekrasov, Seiberg-Witten prepotential from instanton counting, Adv. Theor. Math. Phys. 7 (2003) 831 [hep-th/0206161] [INSPIRE].

[19] N. Nekrasov and A. Okounkov, Seiberg-Witten theory and random partitions, Prog. Math. 244 (2006) 525 [hep-th/0306238] [inSPIRE].

[20] S.-J. Rey and T. Suyama, Exact Results and Holography of Wilson Loops in $N=2$ Superconformal (Quiver) Gauge Theories, JHEP 01 (2011) 136 [arXiv:1001.0016] [INSPIRE].

[21] F. Passerini and K. Zarembo, Wilson Loops in $N=2$ Super-Yang-Mills from Matrix Model, JHEP 09 (2011) 102 [Erratum ibid. 10 (2011) 065] [arXiv:1106.5763] [INSPIRE].

[22] J.G. Russo and K. Zarembo, Localization at Large $N$, in Proceedings, 100th anniversary of the birth of I.Ya. Pomeranchuk (Pomeranchuk 100): Moscow, Russia, June 5-6, 2013, pp. 287-311, arXiv:1312.1214 [INSPIRE].

[23] B. Fiol, E. Gerchkovitz and Z. Komargodski, Exact Bremsstrahlung Function in $N=2$ Superconformal Field Theories, Phys. Rev. Lett. 116 (2016) 081601 [arXiv:1510.01332] [INSPIRE].

[24] M. Baggio, V. Niarchos and K. Papadodimas, $t t^{*}$ equations, localization and exact chiral rings in $4 d \mathcal{N}=2$ SCFTs, JHEP 02 (2015) 122 [arXiv: 1409.4212] [INSPIRE].

[25] M. Baggio, V. Niarchos and K. Papadodimas, On exact correlation functions in $\mathrm{SU}(N)$ $\mathcal{N}=2$ superconformal QCD, JHEP 11 (2015) 198 [arXiv:1508.03077] [INSPIRE].

[26] E. Gerchkovitz, J. Gomis, N. Ishtiaque, A. Karasik, Z. Komargodski and S.S. Pufu, Correlation Functions of Coulomb Branch Operators, JHEP 01 (2017) 103 [arXiv: 1602.05971] [INSPIRE].

[27] M. Billó, F. Fucito, A. Lerda, J.F. Morales, Ya. S. Stanev and C. Wen, Two-point Correlators in $N=2$ Gauge Theories, Nucl. Phys. B 926 (2018) 427 [arXiv:1705.02909] [InSPIRE].

[28] A. Bourget, D. Rodriguez-Gomez and J.G. Russo, A limit for large R-charge correlators in $\mathcal{N}=2$ theories, JHEP 05 (2018) 074 [arXiv:1803.00580] [INSPIRE].

[29] O. Loukas, D. Orlando, S. Reffert and D. Sarkar, An AdS/EFT correspondence at large charge, Nucl. Phys. B 934 (2018) 437 [arXiv: 1804.04151] [inSPIRE].

[30] S. Hellerman, S. Maeda, D. Orlando, S. Reffert and M. Watanabe, Universal correlation functions in rank 1 SCFTs, arXiv:1804.01535 [INSPIRE].

[31] S. Hellerman, D. Orlando, S. Reffert and M. Watanabe, On the CFT Operator Spectrum at Large Global Charge, JHEP 12 (2015) 071 [arXiv:1505.01537] [INSPIRE]. 
[32] L. Álvarez-Gaumé, O. Loukas, D. Orlando and S. Reffert, Compensating strong coupling with large charge, JHEP 04 (2017) 059 [arXiv: 1610.04495] [INSPIRE].

[33] A. Monin, D. Pirtskhalava, R. Rattazzi and F.K. Seibold, Semiclassics, Goldstone Bosons and CFT data, JHEP 06 (2017) 011 [arXiv:1611.02912] [INSPIRE].

[34] S. Hellerman and S. Maeda, On the Large R-charge Expansion in $\mathcal{N}=2$ Superconformal Field Theories, JHEP 12 (2017) 135 [arXiv:1710.07336] [INSPIRE].

[35] O. Loukas, Abelian scalar theory at large global charge, Fortsch. Phys. 65 (2017) 1700028 [arXiv: 1612.08985] [INSPIRE].

[36] S. Hellerman, N. Kobayashi, S. Maeda and M. Watanabe, A Note on Inhomogeneous Ground States at Large Global Charge, arXiv:1705.05825 [INSPIRE].

[37] D. Banerjee, S. Chandrasekharan and D. Orlando, Conformal dimensions via large charge expansion, Phys. Rev. Lett. 120 (2018) 061603 [arXiv:1707.00711] [INSPIRE].

[38] O. Loukas, D. Orlando and S. Reffert, Matrix models at large charge, JHEP 10 (2017) 085 [arXiv: 1707.00710] [INSPIRE].

[39] S. Hellerman, S. Maeda and M. Watanabe, Operator Dimensions from Moduli, JHEP 10 (2017) 089 [arXiv: 1706.05743] [INSPIRE].

[40] D. Jafferis, B. Mukhametzhanov and A. Zhiboedov, Conformal Bootstrap At Large Charge, JHEP 05 (2018) 043 [arXiv: 1710.11161] [INSPIRE].

[41] O. Loukas, A matrix CFT at multiple large charges, JHEP 06 (2018) 164 [arXiv:1711.07990] [INSPIRE].

[42] A.A. Tseytlin, Spinning strings and AdS/CFT duality, hep-th/0311139 [INSPIRE].

[43] S. Cecotti and C. Vafa, Topological antitopological fusion, Nucl. Phys. B 367 (1991) 359 [INSPIRE].

[44] S. Cecotti and C. Vafa, Exact results for supersymmetric $\sigma$ models, Phys. Rev. Lett. 68 (1992) 903 [hep-th/9111016] [INSPIRE].

[45] M. Billó, F. Galvagno, P. Gregori and A. Lerda, Correlators between Wilson loop and chiral operators in $\mathcal{N}=2$ conformal gauge theories, JHEP 03 (2018) 193 [arXiv:1802.09813] [INSPIRE].

[46] M. Buican, T. Nishinaka and C. Papageorgakis, Constraints on chiral operators in $\mathcal{N}=2$ SCFTs, JHEP 12 (2014) 095 [arXiv: 1407.2835] [INSPIRE].

[47] L. Rastelli and S.S. Razamat, The supersymmetric index in four dimensions, J. Phys. A 50 (2017) 443013 [arXiv: 1608.02965] [InSPIRE].

[48] W. Lerche, C. Vafa and N.P. Warner, Chiral Rings in $N=2$ Superconformal Theories, Nucl. Phys. B 324 (1989) 427 [INSPIRE].

[49] E. Gerchkovitz, J. Gomis and Z. Komargodski, Sphere Partition Functions and the Zamolodchikov Metric, JHEP 11 (2014) 001 [arXiv:1405.7271] [INSPIRE].

[50] M. Baggio, V. Niarchos and K. Papadodimas, Exact correlation functions in $\mathrm{SU}(2) \mathcal{N}=2$ superconformal QCD, Phys. Rev. Lett. 113 (2014) 251601 [arXiv:1409.4217] [INSPIRE].

[51] K. Papadodimas, Topological Anti-Topological Fusion in Four-Dimensional Superconformal Field Theories, JHEP 08 (2010) 118 [arXiv:0910.4963] [INSPIRE]. 
[52] S. Penati, A. Santambrogio and D. Zanon, Correlation functions of chiral primary operators in perturbative $N=4 S Y M$, hep-th/0003026 [INSPIRE].

[53] S. Penati, A. Santambrogio and D. Zanon, Two point functions of chiral operators in $N=4$ SYM at order $g^{4}$, JHEP 12 (1999) 006 [hep-th/9910197] [INSPIRE].

[54] S. Penati, A. Santambrogio and D. Zanon, More on correlators and contact terms in $N=4$ SYM at order $g^{4}$, Nucl. Phys. B 593 (2001) 651 [hep-th/0005223] [INSPIRE].

[55] F. Fucito, J.F. Morales and R. Poghossian, Wilson loops and chiral correlators on squashed spheres, JHEP 11 (2015) 064 [arXiv: 1507.05426] [INSPIRE].

[56] D. Rodriguez-Gomez and J.G. Russo, Operator mixing in large $N$ superconformal field theories on $S^{4}$ and correlators with Wilson loops, JHEP 12 (2016) 120 [arXiv:1607.07878] [INSPIRE].

[57] S. Giombi and V. Pestun, Correlators of local operators and $1 / 8$ BPS Wilson loops on $S^{2}$ from 2d YM and matrix models, JHEP 10 (2010) 033 [arXiv:0906.1572] [INSPIRE].

[58] M. Baggio, V. Niarchos, K. Papadodimas and G. Vos, Large- $N$ correlation functions in $\mathcal{N}=2$ superconformal QCD, JHEP 01 (2017) 101 [arXiv:1610.07612] [INSPIRE].

[59] D. Rodriguez-Gomez and J.G. Russo, Large $N$ Correlation Functions in Superconformal Field Theories, JHEP 06 (2016) 109 [arXiv:1604.07416] [INSPIRE].

[60] A. Pini, D. Rodriguez-Gomez and J.G. Russo, Large $N$ correlation functions $\mathcal{N}=2$ superconformal quivers, JHEP 08 (2017) 066 [arXiv: 1701.02315] [INSPIRE].

[61] D.J. Gross and A. Matytsin, Instanton induced large $N$ phase transitions in two-dimensional and four-dimensional QCD, Nucl. Phys. B 429 (1994) 50 [hep-th/9404004] [INSPIRE]. 